\title{
A Multipolar Analysis of Near-Field Absorption and Scattering Processes
}

\author{
Iñigo Liberal, Student Member, IEEE, Iñigo Ederra, Ramón Gonzalo, Member, IEEE, and \\ Richard W. Ziolkowski, Fellow, IEEE
}

\begin{abstract}
A multipolar formulation is adopted to investigate the absorption and scattering processes involved in near-field interactions. This approach allows one to determine the upper bounds for the absorbed and radiated powers that would be achieved by an ideal lossless sensor, which are of particular interest, for example, to wireless power transfer (WPT), wireless sensors and near-field coupled radiators. The multipolar formulation also helps to extricate the fundamental compromises that must be addressed in the design of such systems, as well as to identify strategies that could approach their best possible performances. The general theory is illustrated with an example consisting of a coated sensor illuminated by a Hertzian dipole, which is a representative example of any scattering or radiating system based on small resonators. The example also serves to compare the performance characteristics obtained with different phenomena such as multipolar resonances, phaseinduced interference effects and cloaking.
\end{abstract}

Index Terms-Absorption, near-field interactions, near-field parasitic resonators, scattering, sensors, wireless power transfer

\section{INTRODUCTION}

Whenever a sensor captures power from an electromagnetic field, it also produces a disturbance in the field which is detectable by an external observer. Although this disturbance can be minimized (for example, by using cloaked [1] and forward-scattering [2] sensors), a residual amount of scattered field is produced even with the most careful design. The reason for this is that the power carried by the external field must be reduced by the amount of absorbed power. Therefore, the absorption and scattering processes are intimately correlated, which restricts the power captured by a sensor as a function of the disturbance created in the external field in association with the fields induced within the sensor.

Constrained to far-field (FF) interactions, the correlation between the absorption and scattering processes has been studied by means of the optical theorem [2], multipolar decomposition [3], [4] and equivalent circuit model approaches [4]. All these studies agree that while the ratio of the absorbed to scattered

I. Liberal, I. Ederra and R. Gonzalo are with the Electrical and Electronic Engineering Department, Universidad Pública de Navarra, Pamplona, Spain, e-mail: inigo.liberal@unavarra.es

R. W. Ziolkowski is with the Department of Electrical and Computer Engineering, University of Arizona, Tucson, AZ, 85721 USA, email:ziolkowski@ece.arizona.edu

This work was supported in part by the Spanish Ministry of Science and Innovation, Dirección General de Investigación y Gestión del Plan Nacional de I+D+I, Subdirección General de Proyectos de Investigación, Project Nos. TEC2009-11995 and CSD2008-00066 and by NSF contract number ECCS1126572.

Manuscript received Dec. 22, 2012; revised Apr. 22, 2013 power can be made as large as desired, it comes at the expense of losing absorbed power. In particular, maximization of the absorbed power imposes a constraint, i.e., the equality of the absorbed and scattered powers [3], [4]. In antenna terminology, as the re-radiation from a receiving antenna is diminished, its gain decreases with respect to the maximal antenna gain [5], [6]. This maximum point has been shown to occur when the absorbed and scattered powers are equal [4]. In other words, there is a tradeoff between the visibility and effective area of a receiving antenna.

The scenario is radically different for near-field (NF) interactions, where the external field sources and the sensor are coupled; and thus the power supplied by the sources is altered by the presence of the sensor. Despite this, it is natural to expect that the correlation between absorption and scattering processes also constrains the power balance of these nearfield interactions. As a specific example, the imposition of a given scattered power necessitates a leakage of power from the source-sensor coupled system, which potentially limits the transfer efficiency from the source to the sensor. This paper seeks to investigate this and other aspects when the absorption and scattering processes are correlated under near-field conditions. Subsequently, we will use an inclusive concept of a sensor, i.e., it is both a receiver and a scatterer/radiator of electromagnetic power.

It is worth noting that there are a large number of NF-based technologies. For instance, wireless power transfer (WPT) technologies [7] are required for the development of more efficient charging systems for small electronics and powered implants. Near-field resonant parasitic (NFRP) elements have also been employed to reduce the size and increase the efficiency of electrically small radiators [8]. Furthermore, a number of biological and chemical processes are driven by resonant energy transfer (RET) [9]. Consequently, a comprehensive examination of the associated NF interactions and the correlation of the associated absorption and scattering processes, particularly when small resonators are involved, will lead to a deeper understanding of how to engineer them to optimize the performance of these systems. Naturally, the performance of these systems is also delimited by a large number of technological details, including ohmic losses in the creation of the sources, dissipation in the background medium, efficiency of the receiving antenna and losses in the rectifying circuitry. While particular design guidelines can be derived according to any of these technological aspects (e.g., the selection of the frequency of operation [10] and transmitter current distribution [11] for power transfer in lossy 
tissues), the analysis presented here predicts upper bounds of the performance that are present even when all these issues have been addressed.

A multipolar representation of the power terms is adopted throughout this article. Aside from being a useful tool to compute relevant powers and their limits, this mathematical decomposition also provides insight as a function of the sensor size. For example, the maximal number of interacting multipoles for receiving antennas in FF interactions is set to $N=k_{0} a$ for electrically large antennas and $N=1$ for electrically small antennas, where $a$ is the radius of the smallest sphere circumscribing the antenna [3], [5]. However, care must be exercised in NF interactions even for electrically small sensors, because one is then dealing with the source singularities and a large number of multipoles may be required to obtain good convergence. Furthermore, the sources are modeled as current distributions and the sensor is characterized by its scattering multipolar coefficients. This level of abstraction is convenient to extract conclusions that are independent of the technological details. Related works have also employed a multipolar representation to estimate the efficiency of WPT systems [12], [13]. However, the antenna scattering properties were described based on reciprocity and equivalent antenna circuit models. While equivalent circuits are a useful tool to determine the power at the antenna port, they lack the accuracy to compute the scattered power (see [14] and the references therein). This is due to the fact that, even for small dipole receiving antennas, current distributions different from those of the corresponding transmitters must be considered in the scattering and absorption processes. To avoid such complications and to emphasize the fundamentals, a purely multipolar approach is emphasized in our analysis. This also allows generalizing the results to non-reciprocal devices. Moreover, although the presented upper bounds would be achieved only with ideal lossless antennas/scatterers, the presented formalism is also valid for lossy antennas/scatterers.

The remainder of this article is organized as follows: Section II introduces the geometry of an arbitrary near-field scattering problem and related basic definitions, and Section III formulates the multipolar description of such a problem. Section IV then makes use of this formulation to derive the power and efficiency limits in NF power transfer and scattering/radiating systems, as well as to identify the tradeoffs presented in the design of such systems. The derived theory is illustrated through the analysis of a coated sensor illuminated by a Hertzian dipole in Section V. To finalize, conclusions and discussion of the results are gathered in Section VI.

Time-harmonic $e^{j \omega t}$ field expressions are assumed and omitted hereafter. All power quantities are written as their time-averaged embodiments.

\section{Geometry And Power Definitions}

Fig. 1 depicts the geometry of an arbitrary scattering problem: A given distribution of free electric and magnetic sources $\left(\mathbf{J}_{i}, \mathbf{K}_{i}\right)$, enclosed within a surface $S_{i}$, produces an incident electromagnetic field $\left(\mathbf{E}^{i}, \mathbf{H}^{i}\right)$ that illuminates a scatterer enclosed within a surface $S$. In response to such a field, the

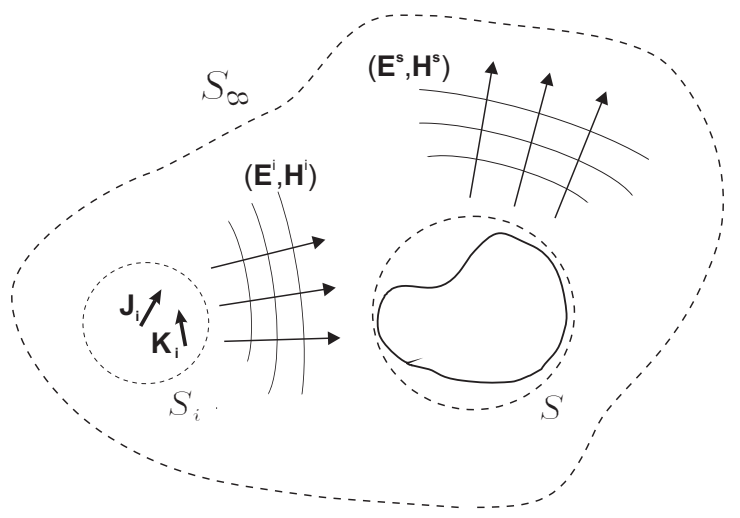

Fig. 1. Sketch of an arbitrary scattering problem. A given distribution of free electric and magnetic sources $\left(\mathbf{J}_{i}, \mathbf{K}_{i}\right)$, enclosed within a surface $S_{i}$, illuminates an arbitrary scatterer enclosed within a surface $S$. The surface $S_{\infty}$ includes both scatterer and source regions.

currents excited inside the scatterer produce a certain scattered field $\left(\mathbf{E}^{s}, \mathbf{H}^{s}\right)$, so that the total field $\left(\mathbf{E}^{t}, \mathbf{H}^{t}\right)$ is equal to the addition of the incident plus scattered fields.

In order to emphasize the absorption and scattering processes produced by the scatterer, it is assumed that both the scatterer and sources are immersed in free-space. In this manner, all the power supplied by the sources, $P_{\text {sup }}$, is either radiated away from the system, $P_{\text {rad }}$, or absorbed inside the scatterer, $P_{\mathrm{abs}}$, i.e.,

$$
P_{\text {sup }}=P_{\text {rad }}+P_{\text {abs }}
$$

In view of (1), efficiencies can be defined to illustrate how the supplied power is divided between the absorption and radiation processes. On the one hand, the power transfer efficiency (PTE), usually employed in WPT analyses, is defined as

$$
\operatorname{PTE}(\%)=\frac{P_{\mathrm{abs}}}{P_{\mathrm{rad}}+P_{\mathrm{abs}}} \cdot 100
$$

Inversely, the radiation efficiency (RE) is defined as

$$
R E(\%)=\frac{P_{\mathrm{rad}}}{P_{\mathrm{rad}}+P_{\mathrm{abs}}} \cdot 100
$$

Both $P_{\text {rad }}$ and $P_{\text {abs }}$ can be determined in terms of the flux of the Poynting vector field through the appropriate surface. On the one hand, $P_{\text {rad }}$ represents the power propagating away from the entire system; and thus it is found as the outward flux of the total Poynting vector field through the surface $S_{\infty}$, which encloses both the source and scatterer regions:

$$
P_{\text {rad }}=P_{S_{\infty}}=\frac{1}{2} \oiint_{S_{\infty}} \operatorname{Re}\left\{\mathbf{E}^{t} \times\left(\mathbf{H}^{t}\right)^{*}\right\} \cdot \widehat{\mathbf{n}} d S
$$

On the other hand, $P_{\text {abs }}$ represents the absorbed power, i.e., the power dissipated within the scatterer; and thus it is found as the inward flux of the total Poynting vector field over a surface $S$, which encloses only the scatterer region:

$$
P_{\mathrm{abs}}=-\frac{1}{2} \oiint_{S} \operatorname{Re}\left\{\mathbf{E}^{t} \times\left(\mathbf{H}^{t}\right)^{*}\right\} \cdot \widehat{\mathbf{n}} d S
$$


Note that, in virtue of Poynting's theorem, $P_{\text {sup }}$ is given by the flux of the total Poynting vector field over a surface enclosing the source region only, e.g., the closed surface $S_{\infty}-S$. Therefore, the definition of the radiated and absorbed powers as given by (4) and (5), respectively, is fully consistent with (1).

It is worth investigating the individual contributions of the incident, scattered and cross-terms to the radiated and absorbed powers. To begin, $P_{\text {rad }}$ can be decomposed as

$$
\begin{array}{r}
P_{\mathrm{rad}}=\frac{1}{2} \oiint_{S_{\infty}} \operatorname{Re}\left\{\mathbf{E}^{i} \times\left(\mathbf{H}^{i}\right)^{*}+\mathbf{E}^{s} \times\left(\mathbf{H}^{s}\right)^{*}+\right. \\
\left.\mathbf{E}^{i} \times\left(\mathbf{H}^{s}\right)^{*}+\mathbf{E}^{s} \times\left(\mathbf{H}^{i}\right)^{*}\right\} \cdot \widehat{\mathbf{n}} d S
\end{array}
$$

Next, the contribution from only the incident field is identified with the power radiated by the free currents, i.e., the power supplied by the sources in the absence of the scatterer:

$$
P_{0}=\frac{1}{2} \oiint_{S_{\infty}} \operatorname{Re}\left\{\mathbf{E}^{i} \times\left(\mathbf{H}^{i}\right)^{*}\right\} \cdot \widehat{\mathbf{n}} d S
$$

Then the contribution from the scattered field is identified with the power radiated only by the scatterer, i.e., the scattered power:

$$
P_{\text {scat }}=\frac{1}{2} \oiint_{S_{\infty}} \operatorname{Re}\left\{\mathbf{E}^{s} \times\left(\mathbf{H}^{s}\right)^{*}\right\} \cdot \widehat{\mathbf{n}} d S
$$

Finally, the contributions from the cross-terms are identified with interference phenomena between the incident and scattered fields. Because of these interference effects, the radiated power can be greater than, equal to or less than the sum of the powers radiated independently by the sources and the scatterer, i.e. $P_{\text {rad }} \lesseqgtr P_{0}+P_{\text {scat }}$. A similar decomposition can be applied to $P_{\text {abs }}$, i.e., to (5). However, since the surface $S$ does not contain any of the sources of the incident field, the contribution from the incident field vanishes in this case, i.e., the power flux from the source fields into and out of $S$ are equal. Contrarily, the sources of the scattered field are fully contained within this surface. Consequently, the contribution from the scattered field only recovers $P_{\text {scat }}$.

As for the cross-terms, recall that according to the optical theorem [15], [16], the extracted power is defined as the sum of the absorbed and scattered powers, i.e., $P_{\text {ext }}=P_{\text {scat }}+P_{\text {abs }}$. Therefore, $P_{\text {ext }}$ is found to be the negative of the flux of the cross-term Poynting vector field over the surface $S$ :

$$
P_{\mathrm{ext}}=-\oiint_{S} \mathbf{S}_{\mathrm{cross}} \cdot \widehat{\mathbf{n}} d S
$$

where

$$
\mathbf{S}_{\text {cross }}=\frac{1}{2} \operatorname{Re}\left\{\mathbf{E}^{i} \times\left(\mathbf{H}^{s}\right)^{*}+\mathbf{E}^{s} \times\left(\mathbf{H}^{i}\right)^{*}\right\}
$$

We emphasize that the flux of $\mathbf{S}_{\text {cross }}$ differs whether it is obtained by integration over the surface $S_{\infty}$ or $S$. In fact, invoking Poynting's theorem to compute $P_{\text {sup }}$ reveals that the difference between both of these flux calculations defines the amount that the power supplied by the sources is altered by the presence of the scatterer, i.e.,

$$
P_{\text {sup }}=P_{0}+\oiint_{S_{\infty}-S} \mathbf{S}_{\text {cross }} \cdot \widehat{\mathbf{n}} d S
$$

\section{Multipolar Representation}

A multipolar representation of the fields is introduced as a tool to compute the various power quantities of interest. It will significantly assist our discussion to elucidate the nearfield balance of powers.

\section{A. Fields Representation}

Outside their respective source regions (for example, outside the surfaces $S_{i}$ and $S$ ), the electromagnetic fields can be decomposed into a series of Tesseral harmonics [6]. In particular, assuming that the origin of the coordinate system is centered within $S$, the fields can be written as superpositions of the Stratton vector fields: $\mathbf{N}_{n m}^{l \lessgtr}$ and $\mathbf{M}_{n m}^{l \lessgtr}$, as

$$
\begin{aligned}
\mathbf{E}^{i} & =\sum_{n=1}^{\infty} \sum_{m=0}^{n} \sum_{l=e, o}\left[-j A_{n m}^{l T M \lessgtr} \mathbf{N}_{n m}^{l \lessgtr}-A_{n m}^{l T E \lessgtr} \mathbf{M}_{n m}^{l \lessgtr}\right] \\
\mathbf{H}^{i} & =\sum_{n=1}^{\infty} \sum_{m=0}^{n} \sum_{l=e, o} \frac{A_{n m}^{l T M \lessgtr} \mathbf{M}_{n m}^{l \lessgtr}-j A_{n m}^{l T E \lessgtr} \mathbf{N}_{n m}^{l \lessgtr}}{\eta_{0}} \\
\mathbf{E}^{s} & =\sum_{n=1}^{\infty} \sum_{m=0}^{n} \sum_{l=e, o}\left[-j B_{n m}^{l T M} \mathbf{N}_{n m}^{l>}-B_{n m}^{l T E} \mathbf{M}_{n m}^{l>}\right] \\
\mathbf{H}^{s} & =\sum_{n=1}^{\infty} \sum_{m=0}^{n} \sum_{l=e, o} \frac{B_{n m}^{l T M} \mathbf{M}_{n m}^{l>}-j B_{n m}^{l T E} \mathbf{N}_{n m}^{l>}}{\eta_{0}}
\end{aligned}
$$

where the $\mathbf{N}_{n m}^{l \lessgtr}$ and $\mathbf{M}_{n m}^{l \lessgtr}$ field vectors are defined as

$$
\begin{aligned}
\mathbf{M}_{n m}^{l \lessgtr}(\mathbf{r}) & =\frac{1}{k_{0}} \nabla \times\left\{\widehat{B}_{n}^{\lessgtr}\left(k_{0} r\right) T_{n m}^{l}(\theta, \phi) \widehat{\mathbf{r}}\right\} \\
& \mathbf{N}_{n m}^{l \lessgtr}(\mathbf{r})=\frac{1}{k_{0}} \nabla \times \mathbf{M}_{n m}^{l \lessgtr}(\mathbf{r})
\end{aligned}
$$

and $k_{0}=\omega \sqrt{\varepsilon_{0} \mu_{0}}$ and $\eta_{0}=\sqrt{\mu_{0} / \varepsilon_{0}}$ denote the free-space propagation constant and impedance, respectively. The $\widehat{B}_{n}^{\lessgtr}(z)$ functions represent the Schelkunoff form of the spherical Bessel functions, with $\widehat{B}_{n}^{<}(z)=\widehat{J}_{n}(z)$ and $\widehat{B}_{n}^{>}(z)=$ $\widehat{H}_{n}^{(2)}(z)$. When used with all of the expressions in this article, the $\lessgtr$ index indicates the representation for the $r<r^{\prime}$ and $r>r^{\prime}$ regions, respectively. This notational choice even for the source field expansion coefficients simplifies the identification of those terms and their corresponding regions in the following discussions. In addition, the $T_{n m}^{l}(\theta, \phi)$ elements are the normalized Tesseral harmonics, given by

$$
T_{n m}^{\left(\begin{array}{l}
e \\
o
\end{array}\right)}(\theta, \phi)=\sqrt{\frac{2 \eta_{0} k_{0}^{2}}{f_{n m}}} P_{n}^{m}(\cos \theta)\left(\begin{array}{l}
\cos (m \phi) \\
\sin (m \phi)
\end{array}\right)
$$

where the constant $f_{n m} \in \mathbb{R}^{+}$is defined as

$$
f_{n m}=\left(1+\delta_{m 0}\right) \frac{2 \pi n(n+1)}{2 n+1} \frac{(n+m) !}{(n-m) !}
$$

and the $P_{n}^{m}(\cos \theta)$ elements denote the associated Legendre polynomials of degree $n$ and order $m$. 


\section{B. Multipolar Coefficients}

The multipolar formulation has been normalized to simplify the power expressions. Therefore, both the source $A_{n m}^{l T Z \lessgtr}$ and scattering $B_{n m}^{l T Z}$ coefficients have been defined with square-root-of-power $(\sqrt{W})$ units. These coefficients are the unknowns of the scattering problem. To start, the source coefficients: $A_{n m}^{l T Z \lessgtr}$, define the excitation. For a generic distribution of free-currents $\left(\mathbf{J}_{i}, \mathbf{K}_{i}\right)$, they can be readily computed by extending the projection approach of Jones [17] to both the electric and magnetic sources

$$
\begin{array}{r}
A_{n m}^{l T M \lessgtr}=\sqrt{\frac{1}{2} \frac{\eta_{0} k_{0}^{2}}{f_{n m}}} \iiint_{V}\left[-\frac{1}{\eta} \mathbf{K}_{i} \cdot \mathbf{M}_{n m}^{l \gtrless}\left(\mathbf{r}^{\prime}\right)\right. \\
\left.-j \mathbf{J}_{i} \cdot \mathbf{N}_{n m}^{l \gtrless}\left(\mathbf{r}^{\prime}\right)\right] d^{3} r^{\prime} \\
A_{n m}^{l T E \lessgtr}=\sqrt{\frac{1}{2} \frac{\eta_{0} k_{0}^{2}}{f_{n m}}} \iiint_{V}\left[\mathbf{J}_{i} \cdot \mathbf{M}_{n m}^{l \gtrless}\left(\mathbf{r}^{\prime}\right)\right. \\
\left.-\frac{j}{\eta} \mathbf{K}_{i} \cdot \mathbf{N}_{n m}^{l \gtrless}\left(\mathbf{r}^{\prime}\right)\right] d^{3} r^{\prime}
\end{array}
$$

On the other hand, determining the scattering coefficients: $B_{n m}^{l T Z}$, involves solving the scattering problem. While they can be determined analytically for a number of canonical shapes (spheres, Rayleigh particles...), in the most general case they are calculated as a post-processing step after a numerical simulation. In particular, once the currents excited in the scatterer have been found by means of a full-wave method, the $B_{n m}^{l T Z}$ can be computed as in (20) and (21), an approach that identifies the currents excited within the scatterer as the sources of the scattered field.

\section{Powers Representation}

Due to the orthogonality of the Tesseral harmonics [6], a total power quantity is equal to the sum of the same power quantity associated with each mode:

$$
P_{\psi}=\sum_{\{q\}} P_{\psi, n m}^{l T Z}
$$

For the sake of simplicity, the sums over all multipoles have been compiled into the multi-index $\{q\}=\{n, m, l, Z\}$, so that

$$
\sum_{\{q\}}=\sum_{n=1}^{\infty} \sum_{m=0}^{n} \sum_{l=e, o} \sum_{Z=E, M}
$$

In this manner, the power terms can be represented in the multipolar expansion as follows

$$
\begin{gathered}
P_{0}=\sum_{\{q\}}\left|A_{n m}^{l T Z>}\right|^{2} \\
P_{\text {scat }}=\sum_{\{q\}}\left|B_{n m}^{l T Z}\right|^{2} \\
P_{\text {rad }}=\sum_{\{q\}}\left|A_{n m}^{l T Z>}+B_{n m}^{l T Z}\right|^{2} \\
P_{\text {ext }}=-\sum_{\{q\}} \operatorname{Re}\left[\left(A_{n m}^{l T Z<}\right)^{*} B_{n m}^{l T Z}\right]
\end{gathered}
$$

$$
P_{\mathrm{abs}}=-\sum_{\{q\}}\left\{\operatorname{Re}\left[\left(A_{n m}^{l T Z<}\right)^{*} B_{n m}^{l T Z}\right]+\left|B_{n m}^{l T Z}\right|^{2}\right\}
$$

\section{Power AND EFFICIENCY Limits}

Because of the interaction terms, the relative locations of the sources and the scatterer significantly impact the various power entities. Consequently, we consider separately the categories of near- and far-field power transfer.

\section{A. Maximizing $P_{\mathrm{abs}}$ : Far-Field Power Transfer}

The sources and the scatterer are effectively decoupled in far-field interactions. Consequently, the power supplied by the sources is independent of the scatterer giving $P_{\text {sup }} \simeq P_{0}$. Intuitively speaking, the incident field decouples from its sources and propagates away to the far zone, where it is intercepted by the scatterer. Even though the scatterer can intercept a power significantly larger than the power illuminating its geometrical cross-section, a finite size scatterer can only absorb a small fraction of the power supplied by the sources. Therefore, the largest $P T E$ is achieved by maximizing $P_{\text {abs }}$, no matter what the re-radiation from the scatterer is.

Inspecting (28), $P_{\text {abs }}$ depends on the scattering coefficients, $B_{n m}^{l T Z}$, as well as the source coefficients in the source region, i.e., $A_{n m}^{l T Z}<$. To maximize the absorbed power for a given source, let us first separate the term inside the brackets into real and imaginary parts, and then take the derivatives with respect to $\operatorname{Re}\left[B_{n m}^{l T Z}\right]$ and $\operatorname{Im}\left[B_{n m}^{l T Z}\right]$. One finally finds that $P_{\text {abs }}$ is maximized for the following condition between the source and scattering coefficients:

$$
B_{n m}^{l T Z}=-\frac{1}{2} A_{n m}^{l T Z<}
$$

Substituting (29) into (28), one finds the maximum absorbed power is given by

$$
\left.P_{\mathrm{abs}}\right|_{\max }=\frac{1}{4} \sum_{\{q\}}\left|A_{n m}^{l T Z}<\right|^{2}
$$

We remark that (30) is valid for scatterers of arbitrary size, shape and constitutive parameters (e.g., non-reciprocal devices). The same limit was independently derived in [3], [4]. Furthermore, introducing (29) into (25), one recovers the known result [2]-[4] that for maximal absorption to occur, the absorbed and scattered powers must be equal, i.e.,

$$
\left.P_{\text {abs }}\right|_{\max }=\left.P_{\text {scat }}\right|_{\max \left\{P_{\text {abs }}\right\}}
$$

Note that $\left.P_{\text {scat }}\right|_{\max \left\{P_{\text {a }}\right\}}$ stands for the scattered power when the absorbed power is maximized. However, this does not correspond to the maximum scattered power. On the other hand, in contrast with the condition of maximal absorption (29), which leads to the equality of absorbed and scattered powers (31), more general ratios of absorbed and scattered powers are possible for alternative relationships between the source $A_{n m}^{l T Z}$ and scattering $B_{n m}^{l T Z}$ coefficients. For example, let us fix $A_{n m}^{l T Z}$ to a given value (i.e., define a given source), and then take the $B_{n m}^{l T Z} \rightarrow 0$ limit (i.e., reduce the response of the scatterer to such incident field). It can be seen by inspecting (25) and (28) that $P_{\text {scat }}$ decreases faster than $P_{\text {abs }}$ 
as $B_{n m}^{l T Z} \rightarrow 0$ and, consequently, arbitrarily large absorbed to scattered power ratios are possible.

Note that $\left.P_{\text {abs }}\right|_{\max }$, as given by (30), is proportional to the magnitude squared of the source coefficients: $\left|A_{n m}^{l T Z}<\right|^{2}$. Since (29) is defined in terms of them, this also means the maximum absorbed power intuitively grows as the source projects a larger field intensity onto the scatterer.

\section{B. Maximizing PTE: Near-Field Power Transfer}

Near-field interactions differ from far-field ones because the scatterer affects the power supplied by the sources, i.e., $P_{\text {sup }} \neq P_{0}$. For example, the interaction between the sources and scatterer can force the sources to increase $P_{\text {sup }}$ to a point where $P_{\text {abs }}$ can be larger than the power supplied by the sources in the absence of the scatterer, $P_{0}$. This is due to the fact that, as illustrated by (31), absorption, scattering and radiation processes are intimately correlated. In view of these correlations, maximizing $P_{\text {abs }}$ does not necessarily lead to the highest $P T E$. For instance, trying to increase $P_{\text {abs }}$ could lead to an uncontrolled leakage of power into the radiated field, which in turn would decrease, not increase, the overall PTE.

Bearing this in mind, the PTE definition, (2), suggests that a strategy to get $100 \%$ power transfer is to suppress $P_{\text {rad }}$ completely. In view of (26), the condition that the multipolar coefficients must satisfy to achieve zero radiated power, $P_{\mathrm{rad}}=0$, is given by

$$
B_{n m}^{l T Z}=-A_{n m}^{l T Z>}
$$

Because $P_{\text {rad }}$ is constructed from the combined radiation coming from the free-sources and the scatterer, the condition (32) represents the case in which both the sources and scatterer are radiating with the same strength $\left(P_{\text {scat }}=P_{0}\right)$ but are outof-phase. In this manner, the fields radiated by the source and scatterer interfere destructively and no net power is radiated away from the system, i.e., $P_{\text {rad }}=0$. It can be checked that (32) is compatible with the presence of absorption. Therefore, there are configurations in which all the power supplied by the sources is absorbed by the scatterer. Moreover, in theory, (32) indeed is compatible with the absorbed power being maximum. Consequently, there are configurations leading to a $100 \%$ PTE, while keeping the absorbed power at a maximum. In particular, this is achieved when the following combined condition is satisfied

$$
B_{n m}^{l T Z}=-A_{n m}^{l T Z>}=-\frac{1}{2} A_{n m}^{l T Z<}
$$

Despite it being an interesting theoretical possibility, one must be cautious about its practicality. In fact, it will be demonstrated in Section V that the combined condition (33) cannot be satisfied by a Hertzian dipole, and therefore it can be inferred that (33) is unlikely to be satisfied with small devices. Moreover, according to (20)-(21), the source coefficients are a strong function of the source location. In particular, (20)-(21) reflect the fact that when the sources are close to the scatterer (i.e., in any near-field interaction), the absolute value of the source coefficients for the $r<r^{\prime}$ region is much larger than the absolute value of the source coefficients in the $r>r^{\prime}$ region, i.e., $\left|A_{n m}^{l T Z}<\right| \gg\left|A_{n m}^{l T Z>}\right|$. Therefore, it can be concluded that (33) also cannot be satisfied in near-field interactions. In other words, it is not possible to simultaneously suppress the radiated power and keep the absorbed power to be at its maximum in near-field interactions.

Let us address the near-field case in more detail. Considering that the scatterer is in the very near-field of the sources $\left(\left|A_{n m}^{l T Z}<\right| \gg\left|A_{n m}^{l T Z\rangle}\right|\right)$, and that there is a significant response to the reactive field $\left(\left|B_{n m}^{l T Z}\right| \gg\left|A_{n m}^{l T Z>}\right|\right)$, the PTE can be approximated as

$$
P T E \simeq \frac{\sum_{\{q\}}\left\{\operatorname{Re}\left[\left(A_{n m}^{l T Z<}\right)^{*} B_{n m}^{l T Z}\right]+\left|B_{n m}^{l T Z}\right|^{2}\right\}}{\sum_{\{q\}} \operatorname{Re}\left[\left(A_{n m}^{l T Z<}\right)^{*} B_{n m}^{l T Z}\right]}
$$

To achieve large power transfer efficiencies in the near-field configurations, asymptotically approaching PTE $=100 \%$, one then finds that it is necessary to enforce the more restrictive coefficient condition

$$
\left|A_{n m}^{l T Z<}\right| \gg\left|B_{n m}^{l T Z}\right| \gg\left|A_{n m}^{l T Z>}\right|
$$

The physical interpretation is as follows. Consider a resonant scatterer placed in the near-field of the sources. It naturally will re-radiate a large amount of power. Thus, the total radiated power is dominated by the scattered power, i.e., $P_{\text {scat }} \gg P_{0}$ so that $P_{\text {rad }} \simeq P_{\text {scat }}$. However, when the losses of the scatterer are tuned to maximize $P_{\text {abs }}$, the absorbed and scattered powers must be equal in accordance with (29). Consequently, half of the supplied power is radiated away. This results in $P T E_{\max }=50 \%$. On the other hand, we note that $P_{\text {abs }} / P_{\text {scat }}$ can be made as large as desired, i.e., $P T E=100 \%$ can be asymptotically approached. However, to resolve the dilemma between these two situations, the latter then has to come at some cost. It is found that this limit can be approached only if the amount of absorbed power is decreased and the corresponding scattered power is decreased even more. Also, consider substituting the far-field condition (29) into (34). This also results in $P T E=50 \%$. This means that the scatterer that maximizes $P T E$ in the far-field case still only can produce $P T E=50 \%$ when it is placed in the very near-field of the sources.

In summary, there is a significant tradeoff between $P T E$ and $P_{\text {abs }}$ in near-field configurations. How then does one optimize a NF-WPT system? First, it was found that maximizing $P_{\text {abs }}$ provides the best PTE in a far-field interaction. In fact, this result is not restricted to only the far field, but it also holds in the intermediate range where $P_{\text {abs }}$ becomes comparable to $P_{0}$. Thus, increasing PTE over $50 \%$ in the near-field can be achieved at the cost of reducing it for larger distances, i.e., the sensor will not have the same PTE in both the near and far field situations. Secondly, hitherto ohmic losses have been disregarded. However, the presence of undesired losses would only decrease the PTE, i.e., they would not be desirable contributions to $P_{\text {abs }}$. Therefore, it can be concluded that maximizing $P_{\text {abs }}$ remains the most robust design against undesired dissipation. Finally, $P_{a b s}$ is defined as the energy per unit time dissipated within the scatterer, which can also be interpreted as the speed at which the energy is transferred from the sources to the scatterer. Undoubtedly, the speed of transfer is a key factor in many WPT systems. 
Since the sources are defined as currents in our analysis, $\left.P_{\text {abs }}\right|_{\max }$ corresponds to the maximal speed of energy transfer per current unit. Therefore, maximizing $P_{\text {abs }}$ also results in the maximal speed of transfer for those systems with a fixed current. In conclusion, the design of WPT systems presents a tradeoff between the PTE in the very near-field case and the $P T E$ as the receiver separates from the sources in the far-field case, as well as the robustness of the system against undesired losses and the speed of transfer for systems with defined currents.

\section{Maximizing $P_{\mathrm{rad}}$ : Near-Field Parasitic Radiators}

Designing an efficient near-field parasitic radiator represents exactly the opposite problem to the WPT case. Naturally, an ideal lossless radiator features $R E=100 \%, R E$ being defined by (3). However, in practice, all materials have some loss, and small radiators are typically inefficient even when they are matched with additional circuitry. Therefore, $P_{\text {rad }}$ must be maximized to achieve an acceptable $R E$ for any radiating system.

Recall that in a near-field configuration, $P_{\text {rad }}$ is constructed from a combination of the fields radiated from both the sources and the scatterer. The multipolar expression of $P_{\text {rad }}$, given by (26), provides a nice intuition on how to maximize it. In particular, applying the triangle inequality to (26), one finds the upper bound on $P_{\mathrm{rad}}$ :

$$
P_{\mathrm{rad}} \leq \sum_{\{q\}}\left(\left|A_{n m}^{l T Z>}\right|+\left|B_{n m}^{l T Z}\right|\right)^{2}
$$

The upper bound is reached whenever the sources and scatterer radiate in-phase, i.e., when $\arg \left\{B_{n m}^{l T Z}\right\}=\arg \left\{A_{n m}^{l T Z}>\right\}$; and when $P_{\text {scat }}$ is maximized. However, inspecting (25) reveals that $P_{\text {scat }}$ grows along with $B_{n m}^{l T Z}$, and thus it cannot be maximized through the same derivation as was used for $P_{\text {abs }}$ in Section IV-A.

Despite this difficulty, an upper bound for the maximum scattered power was derived in [4]. This bound was based on a circuit model approach and is restricted to penetrable scatterers. To assess this bound further, a simpler, albeit more mathematical, derivation is introduced here. To this end, let us assume that the scatterer is passive, linear, and that their surfaces are such that the spherical harmonics interact independently. In such a case, the scattering coefficients are proportional to the source coefficients in the region of the scatterer, i.e.,

$$
B_{n m}^{l T Z}=b_{n m}^{l T Z} A_{n m}^{l T Z<}
$$

and passivity holds independently for each multipole, i.e.,

$$
P_{\mathrm{abs}, n m}^{l T Z}=-\left|A_{n m}^{<l T Z}\right|^{2}\left\{\operatorname{Re}\left[b_{n m}^{l T Z}\right]+\left|b_{n m}^{l T Z}\right|^{2}\right\}>0
$$

Thus, $P_{\text {abs }, n m}^{l T Z}>0$ imposes the conditions: $\operatorname{Re}\left[b_{n m}^{l T Z}\right]<0$ and $\left|\operatorname{Re}\left[b_{n m}^{l T Z}\right]\right| \geq\left|b_{n m}^{l T Z}\right|^{2}$. Furthermore, since $\left|\operatorname{Re}\left[b_{n m}^{l T Z}\right]\right| \leq$ $\left|b_{n m}^{l T Z}\right|, P_{\text {abs }, n m}^{l T Z}>0$ also requires $\left|b_{n m}^{l T Z}\right|^{2} \leq 1$. Therefore, the condition on the $b_{n m}^{l T Z}$ coefficients to get maximal scattered power can be written as

$$
\left|b_{n m}^{l T Z}\right|^{2}=1 \rightarrow b_{n m}^{l T Z}=-1
$$

Therefore, the maximal scattered power is given by

$$
\left.P_{\text {scat }}\right|_{\max }=\sum_{\{q\}}\left|A_{n m}^{l T Z<}\right|^{2}
$$

and this upper bound is obtained when the multipolar coefficients satisfy the relation

$$
B_{n m}^{l T Z}=-A_{n m}^{l T Z<}
$$

After notational changes, (40) recovers the upper bound derived in [4].

Note that the maximum scattered power is four times larger than the maximum absorbed power, i.e. $\left.P_{\text {scat }}\right|_{\max }=$ $\left.4 P_{\mathrm{abs}}\right|_{\max }$. Once the maximum scattered power is known, it is straightforward to derive the maximum radiated power by introducing (41) into (20)-(21). One obtains:

$$
\left.P_{\text {rad }}\right|_{\max }=\sum_{\{q\}}\left(\left|A_{n m}^{l T Z>}\right|+\left|A_{n m}^{l T Z<}\right|\right)^{2}
$$

However, for a NFRP antenna, the scatterer (i.e., the NFRP element) lies in the very near-field of the source (the driven element) giving $\left|A_{n m}^{l T Z<}<\right| \gg\left|A_{n m}^{l T Z>}\right|$. Therefore, the scattered power is dominant over the power radiated by the sources, i.e., $P_{\text {scat }} \gg P_{0}$, and the maximum radiated power can be approximated as

$$
\left.\left.P_{\text {rad }}\right|_{\max } \simeq P_{\text {scat }}\right|_{\max }=\sum_{\{q\}}\left|A_{n m}^{l T Z<}\right|^{2}
$$

This conclusion is in agreement with state-of-the-art NFRP radiator results [8], where most of the radiation comes from the parasitic element rather than the driven element itself.

\section{EXAmple: Hertzian Dipole Illuminating A COATED SENSOR}

We use the canonical problem consisting of a multilayered coated spherical shell excited by an electric Hertzian dipole to clarify the theory and to illustrate how the properties of such a specific configuration approaches the derived limits. Various special cases will be treated in detail. They emphasize the possible extreme, interesting behaviors associated with this configuration. Note that whenever a general source is much smaller than its operational wavelength, its fields are essentially those of an equivalent Hertzian dipole. Therefore this canonical example is representative of a wide range of physical systems.

\section{A. Geometry}

The geometry of this pathfinder example is schematically depicted in Fig. 2. The source consists of an electric Hertzian dipole of current moment $I_{e} l=\left|I_{e} l\right| e^{j \varphi_{I}}$, orientated along the $\widehat{\mathbf{x}}$ direction, and placed on the $-z$ axis at a distance $r^{\prime}$ from the origin of the coordinates. As for the scatterer, it is placed at the origin of the coordinates. It consists of a solid metallic spherical core with conductivity $\sigma_{s}$ and radius $a_{s}$. This core is coated with two material layers. The first layer is a freespace region. The second is a dielectric region, which has the internal and external radii: $a_{1}$ and $a_{2}$, respectively, and whose 


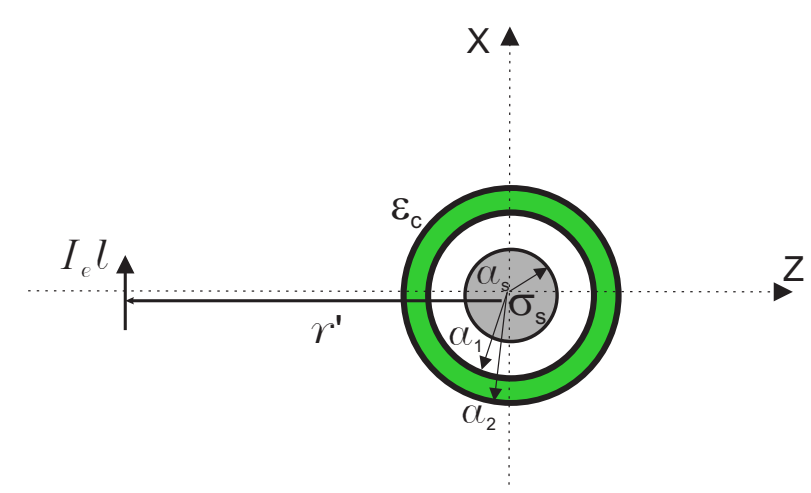

Fig. 2. Sketch of the pathfinder example: an elemental electric Hertzian dipole illuminating a coated sensor.

complex permittivity is $\varepsilon_{c}$. This scatterer will illustrate both the resonant parasitic radiator and general sensor cases.

A perfect electric conducting (PEC, $\sigma_{s} \rightarrow \infty$ ) spherical core will be assumed when the scatterer is acting as a parasitic element. On the other hand, as a sensor, the core will be assumed to have losses associated with its finite conductivity $\sigma_{s}$. These losses will be identified with the power absorbed by the scatterer when it is acting as a sensor, i.e., as the power dissipated in the load of the sensor. This configuration is selected mainly for its mathematical convenience, i.e., spherical shapes can be treated analytically. While actual sensors with electronic loads present generally much more complicated current distributions, this canonical structure will serve to illustrate the previously established limits and to envision potential design guidelines for actual sensors. Specifically, the outer coating layer serves as the means to tune the response of the internal sphere so that it approaches the previously described limits. In fact, it has been studied previously how a coating layer can be employed to enhance the radiation properties of small driven radiators [18], [19]; to hide something inside of it from external observers [1], [20]; and to create resonant scattering configurations [21], [22]. Thus, this example also serves as a means to investigate the properties of such phenomena in NF interactions. Moreover, it has also been demonstrated that the presence of such coatings can be emulated with electrically small NFRP resonators [23] and patterned surfaces [24]. Consequently, the eventual practical challenge is to design structures whose performance characteristics mimic the desired responses obtained from this canonical example. This realizability effort is beyond the scope of the present analysis.

\section{B. Hertzian dipole as a source}

Let us start with the source particulars. For the configuration depicted in Fig. 2, an electric Hertzian dipole (EHD) only produces the $m=1$ and $l=e \mathrm{TM}, l=o$ TE modes, whose coefficients can be found introducing $\mathbf{J}_{i}=I_{e} l \delta\left(\mathbf{r}^{\prime}\right)$ into (20)(21). The latter are:

$$
A_{n 1}^{e T M \lessgtr}=j^{2 n+1} e^{j \varphi_{I}} \sqrt{\frac{3}{4}(2 n+1) P_{0}} \cdot \frac{\partial_{k_{0} r^{\prime}} \widehat{B}^{\gtrless}\left(k_{0} r^{\prime}\right)}{k_{0} r^{\prime}}
$$

$$
A_{n 1}^{o T E \lessgtr}=j^{2 n} e^{j \varphi_{I}} \sqrt{\frac{3}{4}(2 n+1) P_{0}} \cdot \frac{\widehat{B}_{n}^{\gtrless}\left(k_{0} r^{\prime}\right)}{k_{0} r^{\prime}}
$$

where the power supplied to and reradiated by the Hertzian dipole in the absence of the scatterer is [25]

$$
P_{0}=\frac{\eta_{0}}{12 \pi}\left|I_{e} k_{0} l\right|^{2}
$$

As a cross-check to the multipolar formulation, $P_{0}$ is analytically recovered introducing (44)-(45) into (24), and applying the properties of the Bessel function series (10.60.12) and (10.60.14) in [26], [27]. In addition, evaluating the limit $r^{\prime} \rightarrow 0$, only the $n=1$ TM mode survives in the $r>r^{\prime}$ region and it reproduces the well-known fields of a Hertzian dipole located at the origin of the coordinates. In the high-frequency (HF) limit, $k_{0} r^{\prime} \rightarrow \infty$, the scatterer is in the far-field region of the Hertzian dipole and only the $r<r^{\prime}$ region is of interest. Within such a region, we can approximate $\widehat{H}_{n}^{(2)}\left(k_{0} r^{\prime}\right) \simeq$ $j^{n+1} e^{-j k_{0} r^{\prime}}$ and $\partial_{k_{0} r^{\prime}} \widehat{H}_{n}^{(2)}\left(k_{0} r^{\prime}\right) \simeq j^{n} e^{-j k_{0} r^{\prime}}$, so that the TM and TE coefficients are equal, and equal to those of a plane-wave with amplitude

$$
E_{0}=j \frac{\eta_{0} k_{0}^{2}}{4 \pi} I_{e} l \frac{e^{-j k_{0} r^{\prime}}}{k_{0} r^{\prime}}
$$

To finalize the EHD discussion, note that the source coefficients: (44)-(45), in the $r<r^{\prime}$ and the $r>r^{\prime}$ regions differ only by the use of a Hankel or a Bessel function, respectively. This leads to the following interesting property of the source coefficients

$$
\operatorname{Re}\left[A_{n m}^{l T Z>}\left(A_{n m}^{l T Z<}\right)^{*}\right]=\left|A_{n m}^{l T Z>}\right|^{2}
$$

It can be readily checked that condition (33), which describes a simultaneous maximum absorption and a PTE $=100 \%$, is not compatible with condition (48). Therefore, neither Hertzian dipoles nor any small sources approximated by them are capable of supporting simultaneously maximum absorption and PTE $=100 \%$. This fact is true no matter what the distances from the sources to the scatterer are.

\section{Limiting cases with the EHD source}

With these EHD results, the limit of the absorbed power, as given by (30), for a scatterer that is interacting with $N$ multipoles can be approximated in the HF limit as

$$
\left.P_{\mathrm{abs}}^{H F}\right|_{\max } \simeq \frac{3}{8} \frac{P_{0}}{\left(k_{0} r^{\prime}\right)^{2}} \sum_{n=1}^{N}(2 n+1)
$$

Being a purely FF interaction, $P_{\text {abs }}$ decays as $\left(k_{0} r^{\prime}\right)^{2}$ and it is much smaller than the power radiated by the dipole, i.e., $P_{\text {abs }} \ll P_{0}$.

Conversely, the EHD is NF-coupled to the scatterer in the quasi-static (QS) limit: $k_{0} r^{\prime} \rightarrow 0$. Applying the small argument approximations: $\widehat{H}_{n}^{(2)}\left(k_{0} r^{\prime}\right) \simeq j(2 n-1) ! !\left(k_{0} r^{\prime}\right)^{-n}$ and $\partial_{k_{0} r^{\prime}} \widehat{H}_{n}^{(2)}\left(k_{0} r^{\prime}\right) \simeq-j n(2 n-1) ! !\left(k_{0} r^{\prime}\right)^{-(n+1)}$, it is clear that the TM modes dominate over the TE modes, i.e., the reactive electric field is driving the NF interactions. Under 
the QS approximation, the limit of absorbed power can then be approximated as

$$
\left.P_{\mathrm{abs}}^{Q S}\right|_{\max } \simeq \frac{3}{16} P_{0} \sum_{n=1}^{N}\left\{\frac{Q(n)}{\left(k_{0} r^{\prime}\right)^{2 n+4}}+\frac{n^{-2} Q(n)}{\left(k_{0} r^{\prime}\right)^{2 n+2}}\right\}
$$

where

$$
Q(n)=n^{2}(2 n+1) ! !(2 n-1) ! !
$$

and where the first and second addends inside the brackets correspond to the TM and TE modes, respectively. As was anticipated, the TM modes are dominant and thus the reactive electric field is the dominant driving mechanism. In radical opposition to the FF case, (50) reveals that the absorbed power can exceed the power radiated by the free-currents alone by several orders of magnitude, i.e., $P_{\text {abs }} \gg P_{0}$. Consequently, most of the total power supplied by the sources is contributed by the scatterer, i.e., $P_{\text {sup }} \gg P_{0}$. Furthermore, comparing (50) with the multipolar representations of the reactive energy (e.g., [28]), it is revealed that $P_{\text {abs }}$ can also exceed the reactive energy temporarily stored by the sources in the absence of the scatterer. Thus, although the reactive field is the driving mechanism in NF interactions, it is misleading to understand such interactions as a conversion from stored to supplied energies.

It is worth remarking how fast the limit grows along with the multipole order $n$. In view of (50), it can be concluded that the higher-order multipoles can provide substantially larger values of absorbed power. However, in exchange for this behaviour, the higher-order modes decay much faster along with the distance and, for a finite size scatterer, the higher the multipole order, the smaller the bandwidth. Although bandwidth is not a issue for most WPT applications, narrower resonances are harder to tune, and, in the $n \rightarrow \infty$ limit, multipoles with increasingly narrow bandwidths represent diverging transitory times that would never reach stationary state in a practical sense.

Recall that the limit of scattered power is simply four times the limit of the absorbed power, i.e., $\left.P_{\text {scat }}\right|_{\max }=\left.4 P_{\text {abs }}\right|_{\max }$. Therefore, the conclusions drawn for the absorption limits can be directly applied to radiating systems. For example, consider a NFPR antenna that has $P_{\mathrm{rad}} \simeq P_{\text {scat }}$. Thus, the maximal radiated power will be approximately given by four times (50). In addition, it can be similarly concluded that the higher the multipole order, the larger the radiation enhancement and associated cost of bandwidth. This result is in full agreement with the numerical simulations, for instance, in [19], where the radiation from an EHD was enhanced by means of a concentric metamaterial sphere. What is more, our analytical limits make accurate quantitative predictions of the radiation enhancement. In particular, the evaluation of four times (50) with only the $n=1 \mathrm{TM}$ dipolar mode and $r^{\prime}=\lambda / 53.5$, leads to a radiation enhancement of $10 \cdot \log \left(\mathrm{P}_{\mathrm{rad}} / \mathrm{P}_{0}\right) \simeq 59 \mathrm{~dB}$, which can be observed in [19], Fig. 3c. Similarly, taking the $n=2$ quadrupolar TM term only and $r^{\prime}=\lambda / 73.8$, leads to a radiation enhancement of $10 \cdot \log \left(\mathrm{P}_{\mathrm{rad}} / \mathrm{P}_{0}\right) \simeq 107 \mathrm{~dB}$, also in agreement with [19], Fig. 8c.

\section{Lossless Scatterer: Near Field Parasitic Resonator}

Consider now that the EHD is illuminating a lossless scatterer in its near field, i.e., take the scatterer to be a PEC spherical core coated by two lossless dielectric layers. This configuration employs the scatterer as a NFPR element, whose properties can be tailored to those of the Hertzian dipole to achieve various outcomes. To this end, the permittivity of the outer dielectric coating will be tuned to excite different phenomena.

This behavior is evidenced in Fig. 3a, which depicts $P_{\text {rad }}$ and $P_{\text {scat }}$ normalized to $P_{0}$ in a $\mathrm{dB}$ scale as functions of the dielectric constant of the coating, $\varepsilon_{c}$. In order to ensure a strong NF coupling, the EHD is given a current moment $I_{e} k_{0} l=2 \pi \cdot 10^{-4} \mathrm{~A}$ and is placed at a distance $r^{\prime}=0.05 \lambda$ from the origin of the coordinates. The scatterer has an external radius $a_{2}=0.025 \lambda$ and the radii ratios $a_{1} / a_{2}=0.75$ and $a_{s} / a_{2}=0.5$. The spherical expansion was truncated at $N=20$ for all computed examples. This number was heuristically determined, and it has been found to be more than large enough to ensure the convergence of the presented results. To further connect this example to our multipolar formulation, Fig. 3 also depicts the magnitude (Fig. 3b) and phase (Fig. 3c) of the $n=1 \mathrm{TM}$ (electric dipolar) coefficients. Note that each of the vertical lines emphasize permittivities of interest.

The first case of interest is the dark solid line at $\varepsilon_{c} \simeq-0.30$, which coincides with a peak of $P_{\text {rad }}$ that exceeds $P_{0}$ by $35 \mathrm{~dB}$. Therefore, the radiation is mainly driven by the resonator; and, as illustrated in Fig. 3, this point corresponds to the dipole coefficient condition: $B_{11}^{e T M}=-A_{11}^{<e T M}$. In this manner, the limit of radiated power (43) is satisfied. Note that this is in contrast to the exterior region coefficients required in the $P_{\text {rad }}=0$ condition (32). In accordance with (41), the scatterer is acting as a resonator that matches the optimal condition for scattering of the electric dipolar mode. This fact is further illustrated in Fig. 4a, which depicts a contour plot of the total electric field magnitude, $10 \cdot \log _{10}\left|\mathbf{E}^{\mathrm{t}}\right|$, along with the normalized total Poynting vector field, $\mathbf{S}_{\text {tot }}$. The magnitude of the electric field confirms the strong excitation of the electric dipolar mode, and the clearly outgoing Poynting vector field plot is consistent with the fact that the sourcescatterer coupled system is working as a strong radiator. Furthermore, the inset in Fig. $4 \mathrm{a}$ shows the radiation and scattering directivity patterns, $D_{\mathrm{rad}}=r^{2} \widehat{\mathbf{r}} \cdot \mathbf{S}_{\mathrm{tot}} /\left(4 \pi P_{\mathrm{rad}}\right)$ and $D_{\text {scat }}=r^{2} \widehat{\mathbf{r}} \cdot \mathbf{S}_{\text {scat }} /\left(4 \pi P_{\text {scat }}\right)$, respectively, in the xzplane. The dipolar excitation is ratified as both the radiated and scattering directivities have a purely dipolar form, with $D_{\max }=1.5(1.62 \mathrm{~dB})$.

Fig. 4 also allows one to examine the contributions to the total power flux from its incident, scattered, and cross-terms components. From (7) and (8) it is clear that the incident and scattered Poynting vector fields, $\mathbf{S}_{\mathrm{i}}$ and $\mathbf{S}_{\mathrm{s}}$, represent the radiation from the sources and scatterer, respectively. In contrast, the cross-terms Poynting vector field, $\mathbf{S}_{\text {cross }}$, represents the power flow based on the interference between the incident and scattered fields [29]. In this manner, the combined incident plus cross-terms Poynting vector field illustrates how the 


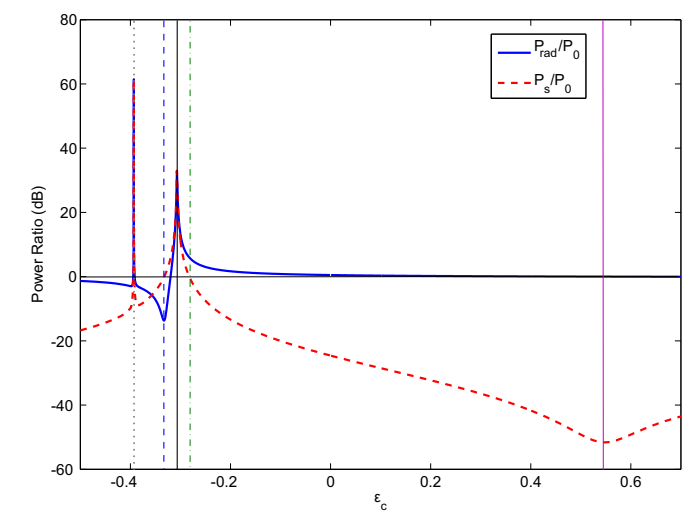

(a)

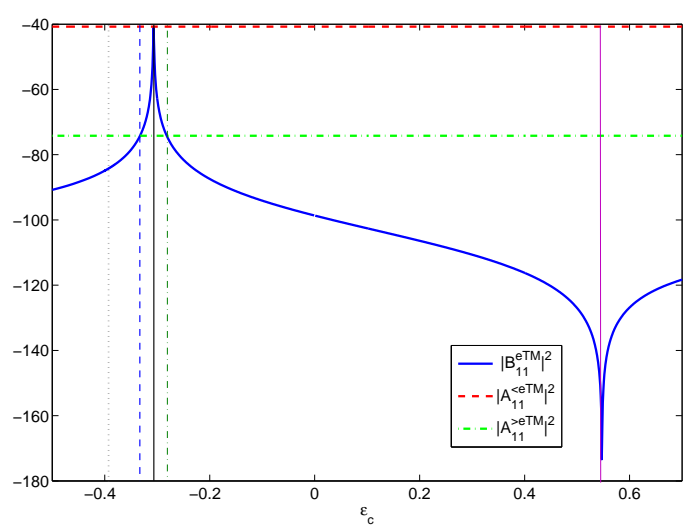

(b)

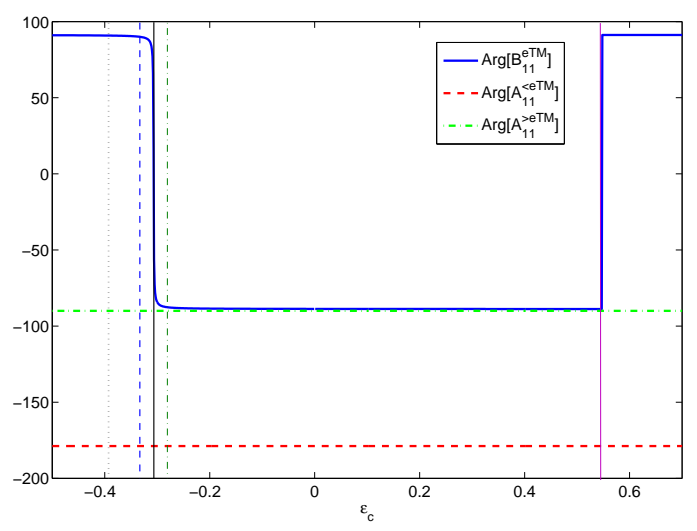

(c)

Fig. 3. (a) Radiated $P_{\text {rad }}$ and scattered $P_{\text {scat }}$ powers, normalized with respect to the power radiated by the sources $P_{0}$, as a function of the dielectric constant of the outer coating layer $\varepsilon_{c}$. (b) Magnitude and (c) phase of the source $\left(A_{11}^{<e T M}\right.$ and $\left.A_{11}^{>e T M}\right)$ and scattering $\left(B_{11}^{e T M}\right)$ electric dipolar coefficients as functions of the dielectric constant of the outer coating layer $\varepsilon_{c}$.

incident field interacts with the scatterer [30]. This combined vector field is depicted in Fig. 4b. As the radiation from the source-scatterer system is dominated by the scattered field, $P_{\text {rad }} \simeq P_{\text {scat }}$, the combined incident plus cross-terms Poynting vector field plot does not present an outgoing trend. Instead,

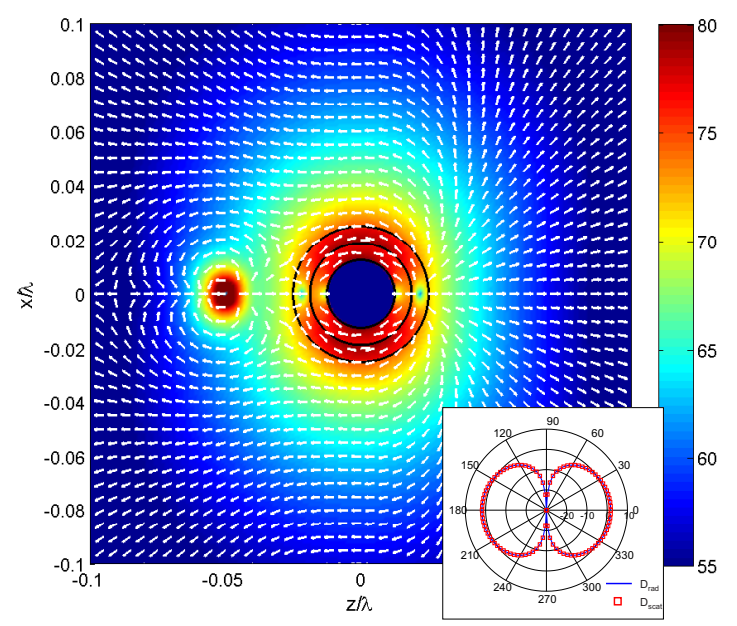

(a)

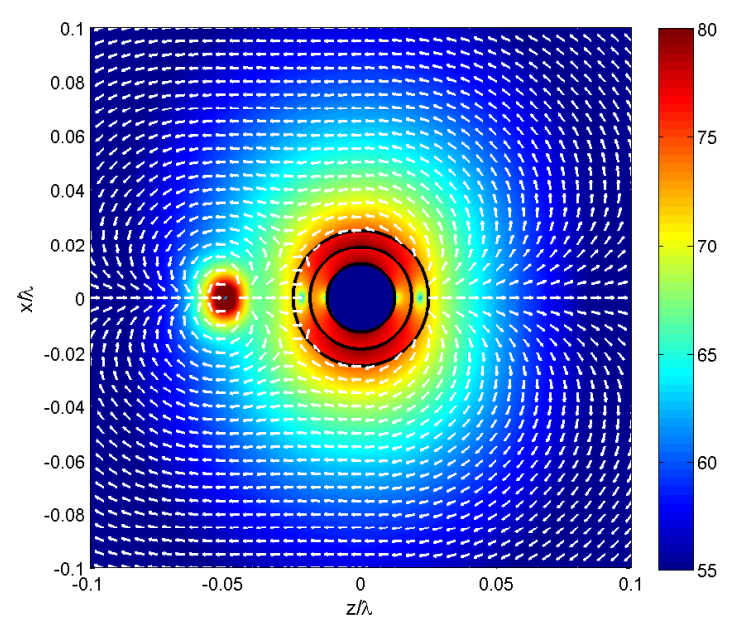

(b)

Fig. 4. [Color online] Contour plot at the dipolar resonance $\left(\varepsilon_{c} \simeq-0.30\right)$ of the magnitude of the total electric field, $10 \cdot \log _{10}\left|\mathbf{E}^{\mathrm{t}}\right|$, along with the (a) normalized total Poynting vector field, $\mathbf{S}_{\text {tot }}$, and (b) incident plus crossterms combined Poynting vector field, $\mathbf{S}_{\mathrm{i}}+\mathbf{S}_{\text {cross }}$. Bottom-right inset of (a): Scattering and radiation directivities.

the power flows from the source to the scatterer, and then it is scattered back to the source. We interpret this feedback as part of the mechanism through which the EHD source is forced to supply more power because of the presence of the scatterer (and, ultimately, the action of the scattered field over the source region).

The second case is the grey-dotted line in Fig. 3, which is specified by $\varepsilon_{c} \simeq-0.39$. It indicates another peak of the radiated power: one for which $P_{\mathrm{rad}}$ is larger than $P_{0}$ in excess of $60 \mathrm{~dB}$. In contrast to the dipole case, no particular behaviour is observed in Figs. 3b and 3c. This is due to the fact that the peak corresponds to the optimal excitation of the $n=2 \mathrm{TM}$ (electric quadrupolar) mode, i.e., $B_{21}^{e T M}=-A_{21}^{<e T M}$. The dominant role of the quadrupolar mode is evidenced in Fig. 5, where both the magnitude of the electric field and the total Poynting vector field reveal clear quadrupolar radiation. Again, the inset of the figure represents the corresponding radiation 


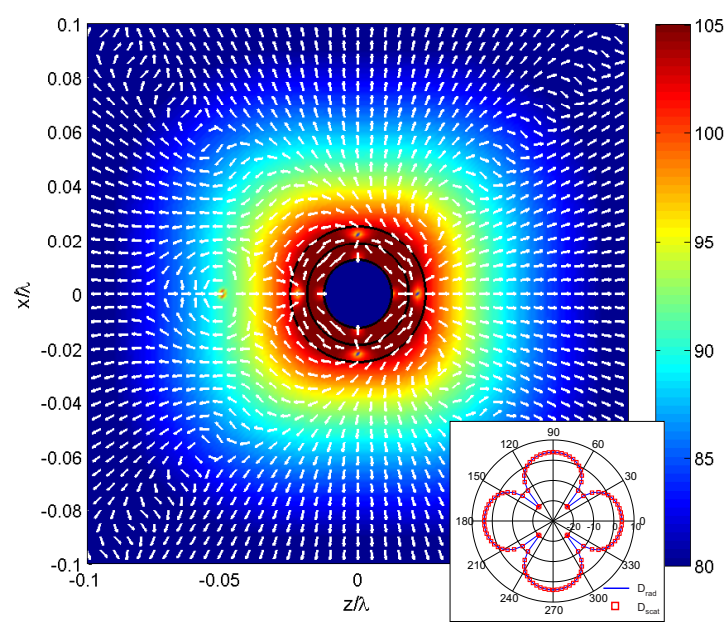

Fig. 5. [Color online] Contour plot at the quadrupolar resonance $\left(\varepsilon_{c} \simeq\right.$ -0.39 ) of the magnitude of the total electric field, $10 \cdot \log _{10}\left|\mathbf{E}^{\mathrm{t}}\right|$, along with the normalized total Poynting vector field, $\mathbf{S}_{\text {tot }}$. Bottom-right inset: Scattering and radiation directivities.

and scattering directivities. Despite the fact that the source is radiating a dipolar mode, the radiation from the scatterer is so overwhelming dominant, i.e., $P_{\text {scat }} \gg P_{0}$, that both the radiation and scattering directivies feature a purely quadrupolar mode whose maximum directivity is $D_{\max }=3(4 \mathrm{~dB})$.

Note that both the dipolar and quadrupolar examples satisfied (41). Therefore, it can be concluded that the presented scatterer is able to reach the limits of the maximum scattered and (for all practical purposes) radiated powers. As expected, the quadrupolar excitation produces a stronger but narrower resonance than the dipolar one.

Aside from multipolar resonances, there are two cases of interest adjacent to the dipole value. Both the blue-dashed $\left(\varepsilon_{c}=-0.33\right)$ and the green-dashed-dotted $\left(\varepsilon_{c}=-0.28\right)$ lines mark permittivities at which the scattered power is equal to the power radiated by the free currents, i.e., $P_{\text {scat }}=P_{0}$. However, while the former reduces $P_{\text {rad }}$ to $14 \mathrm{~dB}$ below $P_{0}$, the latter increases $P_{\text {rad }}$ to four times ( $6 \mathrm{~dB}$ above) its value. Figs. $3 \mathrm{~b}$ and $3 c$ also reveal that at $\varepsilon_{c}=-0.33$ the scatterer is radiating out-of-phase with the source. Thus, the power radiated that is associated with the dipolar mode is reduced by means of destructive interference between the fields generated by the EHD and the scatterer, i.e., $B_{11}^{e T M} \simeq-A_{11}^{>e T M}$ following the condition (32). In contrast, the source and scatterer are radiating in-phase at $\varepsilon_{c}=-0.28$. As a result, the $6 \mathrm{~dB}$ enhancement of $P_{\text {rad }}$ corresponds to the coherent addition of incident and scattered fields, i.e., $B_{11}^{e T M} \simeq+A_{11}^{>e T M}$. In other words, the excitation of the scatterer in the out-of-phase (inphase) case corresponds to it acting as though it were the image of the source that would be formed if there were a PEC (PMC) wall between the source and the scatterer.

The difference between out-of-phase and in-phase cases is also observed at the field level. For example, Fig. 6 depicts the magnitude of the electric field for both the out-of-phase (Fig. 6a) and in-phase (Fig. 6b) cases. It can be concluded that while the strength of the NF electric field is comparable in both cases, the strength of the electric field at a distance from the sources-scatterer system is substantially smaller in the out-of-phase case. In order to emphasize the interference phenomena, Fig. 6 also includes the normalized $\mathbf{S}_{\text {cross }}$ vector field. Since $\mathbf{S}_{\text {cross }}$ represents the interference power flow due to the interaction between the incident and scattered fields, the flux of power carried by the cross-terms is incoming for the out-of-phase case (Fig. 6a), i.e., the interference reduces the outgoing flux. In contrast, the flux of power carried by the cross-terms is outgoing for the in-phase case (Fig. 6b), i.e. the interference increases the outgoing flux. Moreover, the difference between the out-of-phase and in-phase cases is also observed in the radiation and scattering directivities (see the insets of Fig. 6). On the one hand, the dipolar radiation from the scatterer weakens the overall dipolar radiation in the out-of-phase case. Therefore, while the scattering directivity is dipolar, the radiation directivity is a combination of the dipolar and quadrupolar modes. In contrast, the dipolar radiation from the source is reinforced by the scatterer in the in-phase case. Thus, both the scattering and radiation directivities are purely dipolar.

The fifth and final case is given by the purple-solid line $\left(\varepsilon_{c} \simeq 0.545\right)$ in Fig. 3. It marks the point of minimum scattered power and is usually labelled as the cloaking and/or scattering cancellation case. More specifically, Fig. $3 \mathrm{~b}$ connects this point to a vanishing dipolar scattering coefficient, i.e., $B_{11}^{e T M} \simeq 0$. The TE and higher-order TM multipoles produce a residual scattering $50 \mathrm{~dB}$ below $P_{0}$, the power radiated by the free currents. Consequently, its behavior can be fairly approximated as $P_{\text {scat }} \simeq 0, P_{\text {rad }} \simeq P_{0}$. This is illustrated in Fig. 7, which represents the contour plot of the magnitude of the electric field and the normalized $\mathbf{S}_{\text {tot }}$ vector field. Despite the fact that fields are indeed excited within the scatterer and that the power effectively flows within it, there is little to no disturbance on the field or the flux of power produced by the source. It can be concluded that, aside from the known FF configurations [1], it is also possible to place a cloaked scatterer in the NF region of the sources, while keeping it hidden from external observers [31]. The directivity patterns placed on the inset of Fig. 7 further reaffirm this conclusion. While the scattering directivity is constructed from a combination of the TE and higher-order TM multipoles, the contribution of the scattered power to the radiated power is so small that the radiation directivity is that of the EHD alone. Note that it has been argued that, in accordance to the optical theorem, the scattering directivity of a cloaked sensor is that of a forward-scattering sensor [1]. This fact is jeopardized in the NF case because there is not a uniquely defined forward-scattering direction. As a matter fact, if the source is moved to be $10 \lambda$ from the scatterer, the expected forward-scattering directivity (towards the $+z$ axis), as shown in the top-right inset of Fig. 7, is recovered.

\section{E. Lossy Scatterer: Near Field Wireless Power Transfer}

As losses are introduced within the scatterer, the power supplied by the sources is not fully radiated, but rather divided between absorption and radiation. For example, when the internal sphere has a finite conductivity, $\sigma_{s}$, the electromagnetic field penetrates inside it, and a certain amount of 


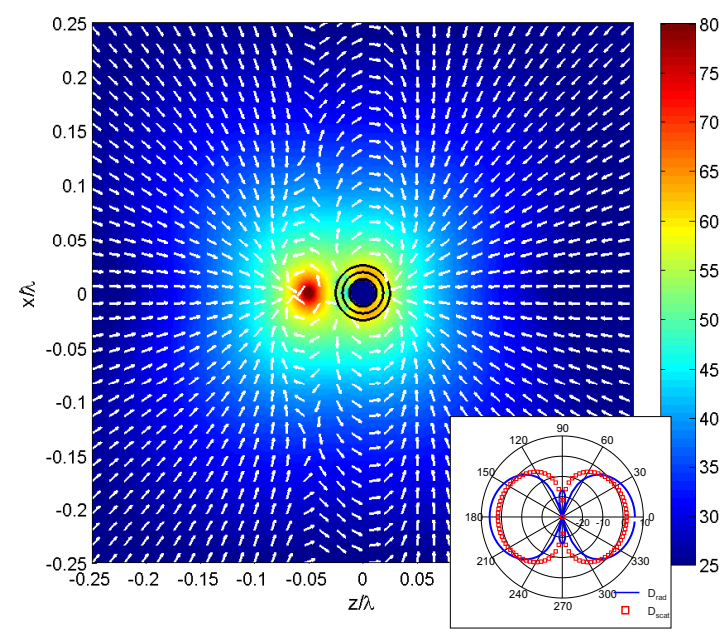

(a)

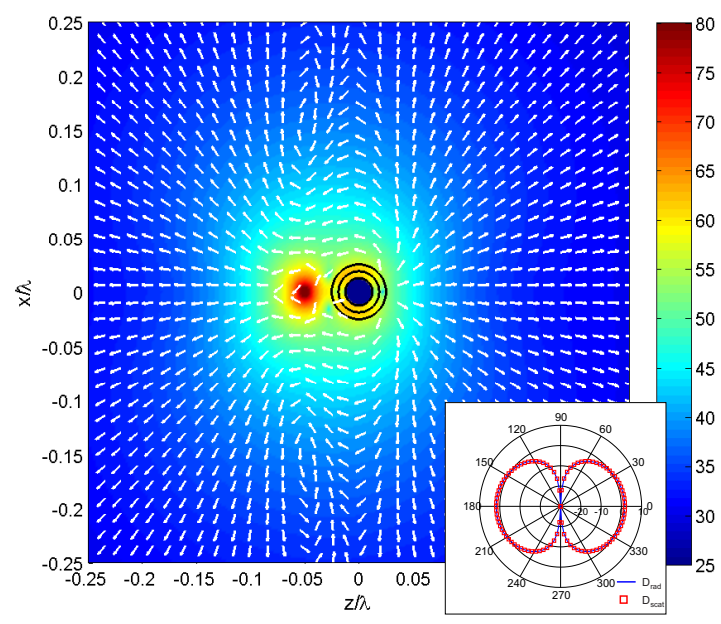

(b)

Fig. 6. [Color online] Contour plot at the (a) out-of-phase $\left(\varepsilon_{c} \simeq-0.33\right)$ and (b) in-phase $\left(\varepsilon_{c} \simeq-0.28\right)$ cases, of the magnitude of the total electric field, $10 \cdot \log _{10}\left|\mathbf{E}^{\mathrm{t}}\right|$, along with the normalized cross-terms Poynting vector field, $\mathbf{S}_{\text {cross }}$. Bottom-right insets: Scattering and radiation directivities.

power is dissipated in the creation of conduction currents. For illustrative purposes, this absorption mechanism will be considered to exemplify the performance of the scatterer as a sensor/NF WPT receiver. In this manner, the power dissipated within the sphere will be considered as the power captured by the sensor, and it will be the baseline to determine $P_{\text {abs }}$ and PTE. This section studies the evolution of the power balance as the medium resistance of the internal sphere, i.e., $\operatorname{Re}\left[\eta_{\mathrm{s}}\right]$, where $\eta_{s}=\sqrt{j \omega \mu_{0} / \sigma_{s}}$, increases. In other words, the evolution of the power balance as the sphere conductivity decreases, and the electromagnetic fields are allowed to penetrate more completely inside the sphere. The selection of plotting the quantities of interest against the term $\operatorname{Re}\left[\eta_{\mathrm{s}}\right]$, rather than simply $\sigma_{s}$, is made purely for illustrative purposes. In particular, this choice avoids abrupt changes in the curves and also incorporates the frequency.

Fig. 8 illustrates how the radiated, scattered and absorbed

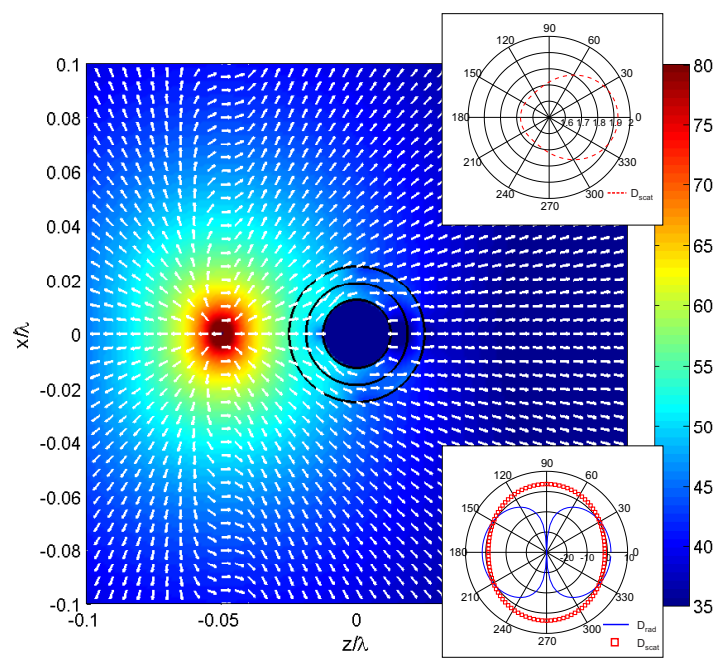

Fig. 7. [Color online] Contour plot at the cloaking point $\left(\varepsilon_{c} \simeq 0.55\right)$ of the magnitude of the total electric field, $10 \cdot \log _{10}\left|\mathbf{E}^{\mathrm{t}}\right|$, along with the normalized total Poynting vector field, $\mathbf{S}_{\text {tot }}$. Bottom-right inset: Scattering and radiation directivities. Top-right inset: Scattering directivity at $r^{\prime}=10 \lambda$

powers (Fig. 8a), as well as the PTE (i.e., $P_{\text {abs }}$ normalized by $P_{\text {sup }}$, Fig. $8 \mathrm{~b}$ ), for the dipolar resonance evolve along with the medium resistance of the metallic sphere. It can be concluded that $P_{\text {rad }}$ and $P_{\text {scat }}$ monotonically decrease along with the medium resistance. By contrast, $P_{\mathrm{abs}}$ is maximized for a specific medium resistance. As demonstrated in Section IV, the maximum absorbed power is $6 \mathrm{~dB}$ (4 times) smaller than the maximum scattered power. Furthermore, the condition (31) holds at the point of maximum absorption, i.e., where $P_{\text {abs }}=P_{\text {scat }}$. Since the scattered and radiated powers are approximately equal, the $P T E$ at the point of maximum absorption is $50 \%$. Note also that while $P_{\text {abs }}$ is reduced along with the medium resistance, the absorbed-to-scattered power ratio increases monotonically, as does the PTE. Therefore, as noted previously, high PTEs are obtained at the cost of reducing $P_{\text {abs }}$.

It is interesting to compare the cases of maximizing $P_{\text {abs }}$ and PTE at the field level. To this end, Fig. 9 gives the contour plot of the magnitude of the electric field along with the normalized total Poynting vector field at the dipolar resonance $\left(\varepsilon_{c} \simeq-0.30\right)$. Specifically, Fig. 9a presents this behavior when the medium resistance $\operatorname{Re}\left[\eta_{s}\right]=9.6 \Omega$, which maximizes $P_{\text {abs }}$. On the other hand, Fig. $9 \mathrm{~b}$ presents it when the medium resistance $\operatorname{Re}\left[\eta_{s}\right] 49.8 \Omega$ and the power transfer is highly efficient: $P T E=95 \%$. Comparing the two figures, one finds that the electric field excited by the scatterer for resistance yielding the maximum absorption is significantly stronger than the one corresponding to the efficient power transfer. This weakening of the electric field in the latter case is consistent with the reduction of $P_{\text {rad }}$ and $P_{\text {scat }}$ observed in Fig. 8. Moreover, the total Poynting vector field presents a clear outgoing trend for the maximum absorbed power case, which is consistent with a leakage of power that reduces the transfer efficiency. In contrast, the outgoing flux of power collapses when the medium resistance and, therefore, the transfer efficiency is increased. In such a case, no net power 


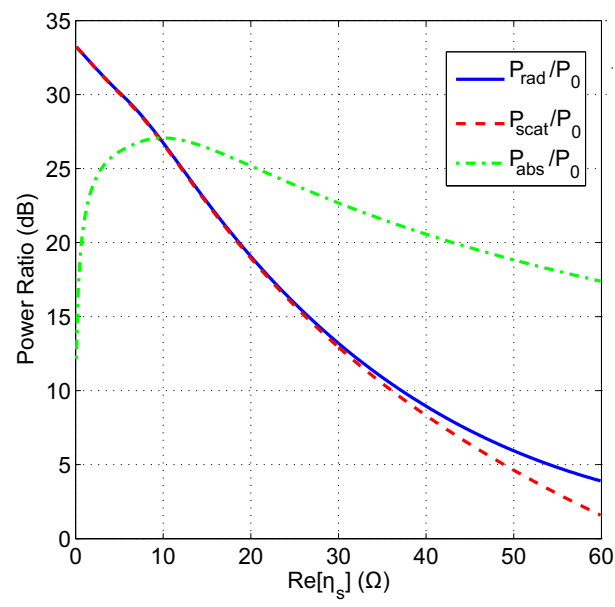

(a)

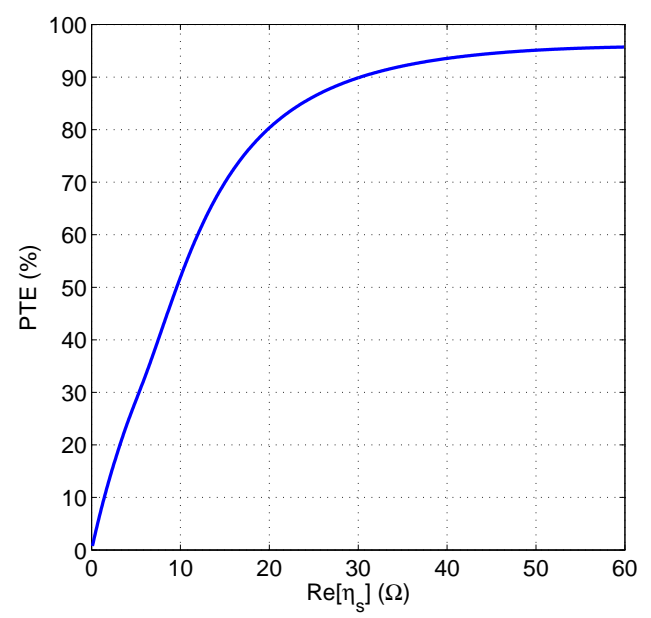

(b)

Fig. 8. (a) Power ratios and (b) power transfer efficiency (PTE) at the dipolar resonance $\left(\varepsilon_{c} \simeq-0.30\right)$ as functions of the medium resistance, $\operatorname{Re}\left[\eta_{s}\right]$, in the metallic spherical core.

is escaping the system. The streamlines of the power flux are found to conform to closed paths between the source and the scatterer.

As it was demonstrated in Section IV, decreasing the absorbed power deteriorates the transfer efficiency at larger distances. This is illustrated in Fig. 10, which presents the $P T E$ as a function of the source-scatterer separation, $k_{0} r^{\prime}$. As representative samples, the figure includes the curves for medium resistances equal to $\operatorname{Re}\left[\eta_{s}\right]=4.2 \Omega, \operatorname{Re}\left[\eta_{s}\right]=9.6 \Omega$, $\operatorname{Re}\left[\eta_{s}\right]=19.8 \Omega$ and $\operatorname{Re}\left[\eta_{s}\right]=48.9 \Omega$. These values correspond to PTEs in the NF configuration equal to $25 \%$ $50 \%, 80 \%$ and $95 \%$, respectively. As expected, the best PTE, when the source separates from the scatterer, is obtained for a medium resistance $\operatorname{Re}\left[\eta_{s}\right]=9.6 \Omega$. Again, this is the medium resistance that maximizes $P_{\text {abs }}$ in Fig.8.

Similarly, Fig. 11 presents how the radiated, scattered and absorbed powers evolve along with the medium resistance in the metallic spherical core for the quadrupolar resonance case $\left(\varepsilon_{c} \simeq-0.39\right)$. The power ratios are given in Fig. 11a and the PTE values in Fig. 11b. The behaviors are essentially the

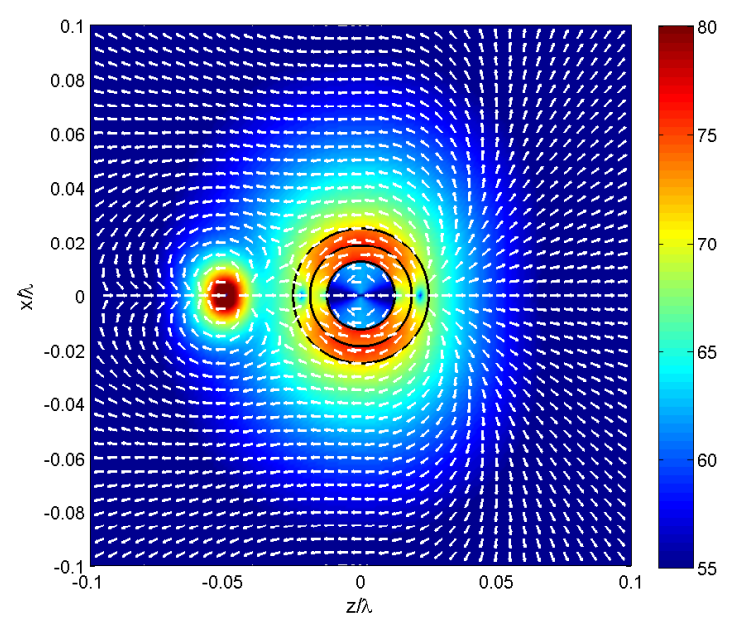

(a)

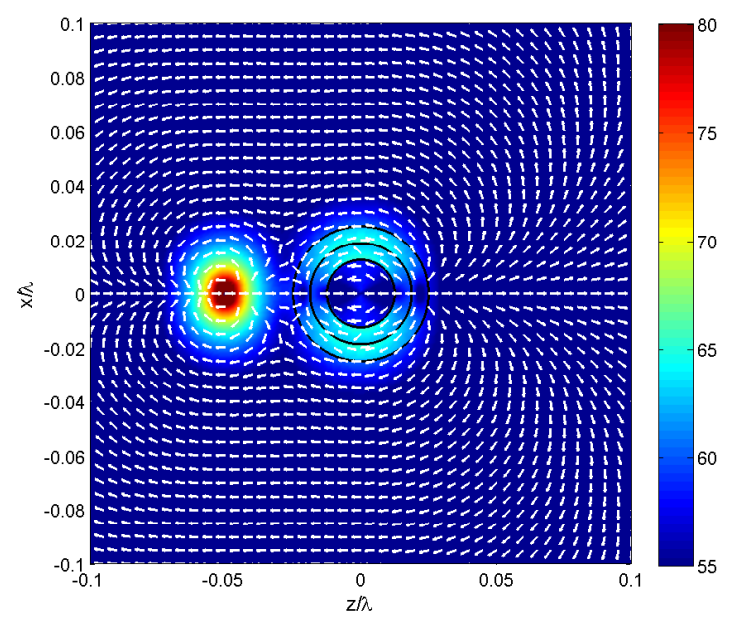

(b)

Fig. 9. [Color online] Contour plot at the dipolar resonance $\left(\varepsilon_{c} \simeq-0.30\right)$ of the magnitude of the total electric field, $10 \log _{10}\left|\mathbf{E}^{\mathrm{t}}\right|$, along with the normalized total Poynting vector field, $\mathbf{S}_{\text {tot }}$. Medium resistances in the metallic spherical core are (a) $\operatorname{Re}\left[\eta_{s}\right]=9.6 \Omega$ and (b) $\operatorname{Re}\left[\eta_{s}\right]=49.8 \Omega$.

same as those found in the dipolar case, but the quadrupole resonance values are much more sensitive to the loss effects and the values of $P_{\text {abs }}$ are significantly larger.

Because of the notable differences in the maxima associated with the different modes, the convenience of exciting the dipolar, quadrupolar or even higher order resonances in a small resonator must be discussed. To further clarify this, Fig. 12 depicts $P_{\text {abs }}$ and PTE for the dipolar and quadrupolar resonance cases as functions of the source-scatterer electrical separation distance when the medium resistances are set to their respective values that maximize the absorbed power. From these results, it can be established that the quadrupolar case provides better $P_{\text {abs }}$ and $P T E$ values in both the NF and FF interactions, as well as a comparable performance at intermediate distances. This is due to the fact that in the NF limit: $k_{0} r^{\prime} \ll 1$, the interactions are stronger the higher the multipole order, as exemplified by (50). Furthermore, 


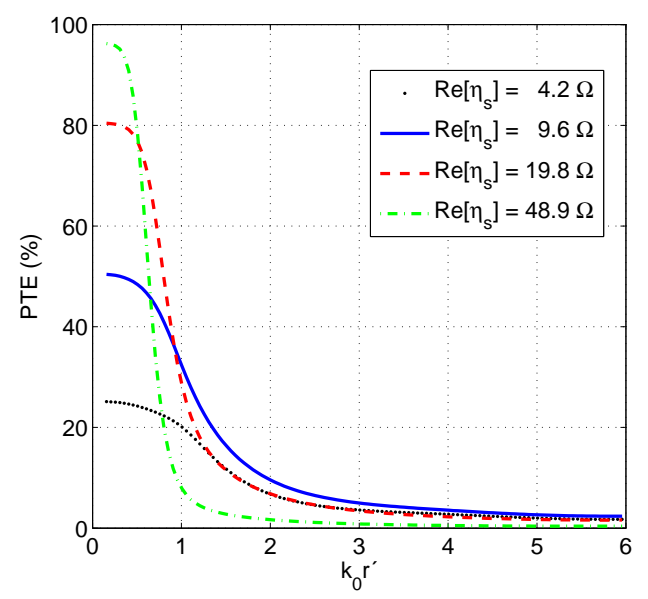

Fig. 10. Power transfer efficiency PTE at the dipolar resonance $\varepsilon_{c} \simeq-0.30$ as a function of the source-sensor electrical separation distance, $k_{0} r^{\prime}$, for a metallic spherical core with medium resistances equal to $\operatorname{Re}\left[\eta_{s}\right]=4.2 \Omega$ $\operatorname{Re}\left[\eta_{s}\right]=9.6 \Omega, \operatorname{Re}\left[\eta_{s}\right]=19.8 \Omega$ and $\operatorname{Re}\left[\eta_{s}\right]=48.9 \Omega$.

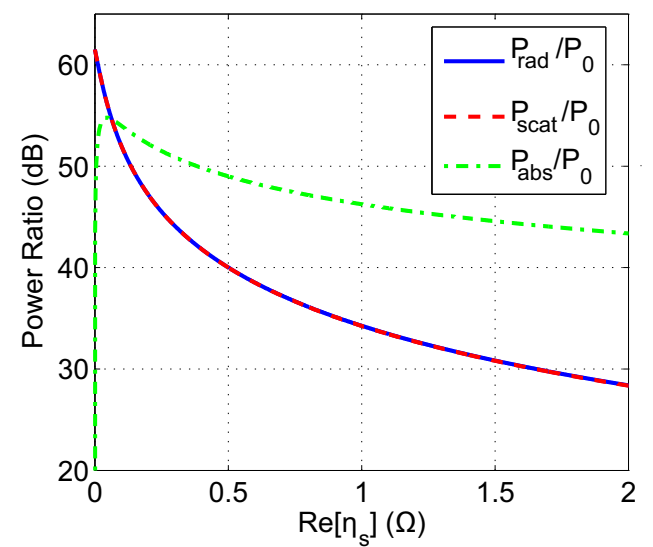

(a)

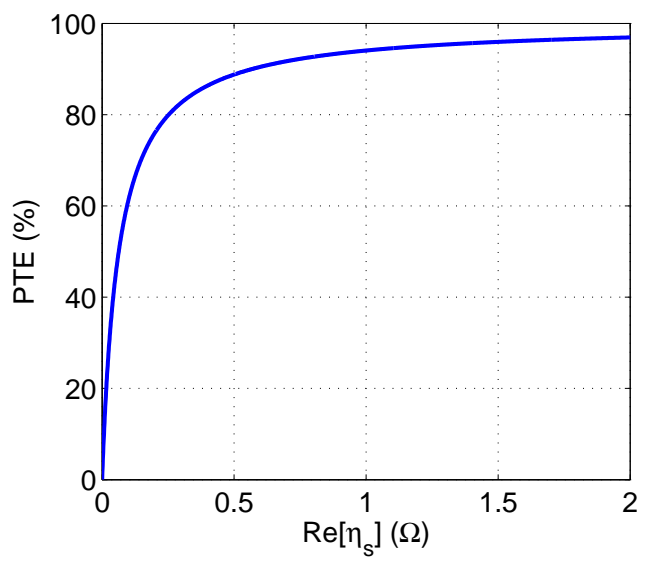

(b)

Fig. 11. (a) Power ratios and (b) power transfer efficiency PTE at the quadrupolar resonance $\left(\varepsilon_{c} \simeq-0.39\right)$ as functions of the medium resistance, $\operatorname{Re}\left[\eta_{s}\right]$, in the metallic spherical core.

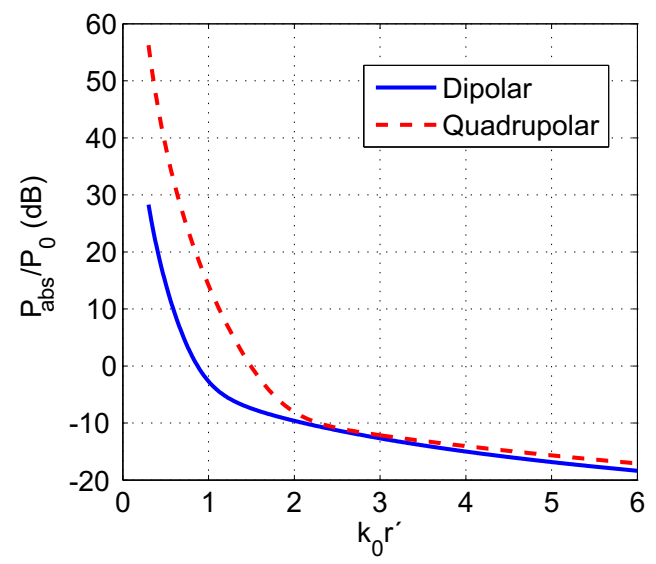

(a)

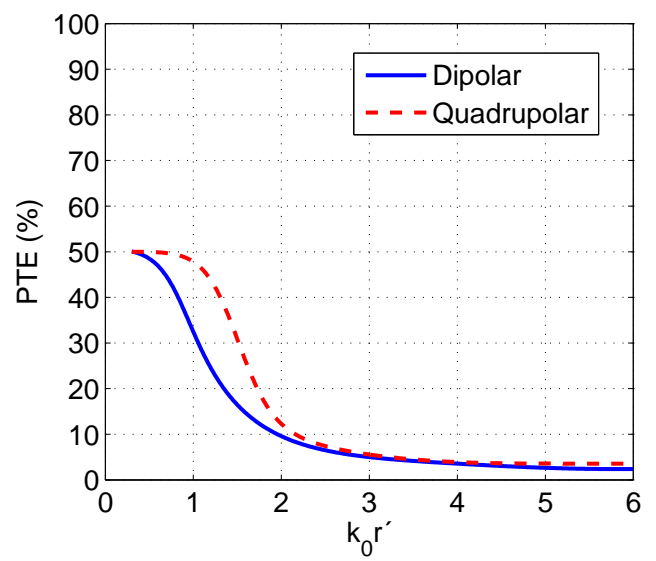

(b)

Fig. 12. Comparion of (a) Power ratios and (b) power transfer efficiency $P T E$ for dipolar and quadrupolar resonant scatterers as functions of the source-scatterer electrical separation distance, $k_{0} r^{\prime}$.

for the FF interactions, where $k_{0} r^{\prime} \gg 1$, the effective area grows along with the multipole order, as shown in (49). At intermediate distances, i.e., between the NF and FF interaction distances, the NF contributions of the higher multipole orders decay faster, and the performance of the multipoles becomes comparable. Despite these results, it cannot be overlooked that the bandwidth is successively smaller as the multipole order becomes larger. Note, however, that bandwidth is not a stringent requirement in WPT technologies. As a matter of fact, a narrow bandwidth is convenient to avoid interferences with other wireless systems. Nevertheless, an excessively narrow bandwidth is much more difficult to accommodate in practice and extra energy will be wasted in a larger transitory time. An additional drawback of the quadrupolar and higher order resonances is, as shown in Fig. 11, that they are much more sensitive to absorption. Therefore, undesired dissipation in the absorber could overdamp the resonance and detune it, losing any anticipated advantages.

As it was pointed out in Section IV, an attractive strategy to obtain a highly efficient WPT system is to employ a scatterer that is radiating out-of-phase with respect to the sources. In this regard, Fig. 13 presents the out-of-phase case $\left(\varepsilon_{c} \simeq-033\right)$ evolution of the radiated, scattered and absorbed 


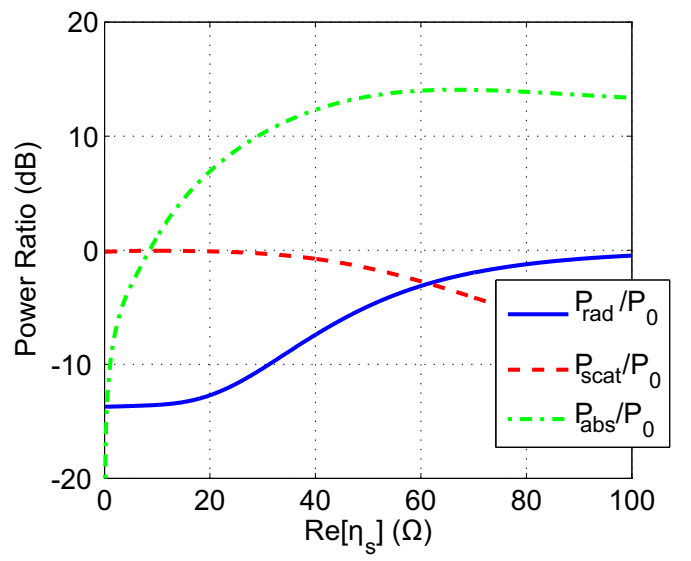

(a)

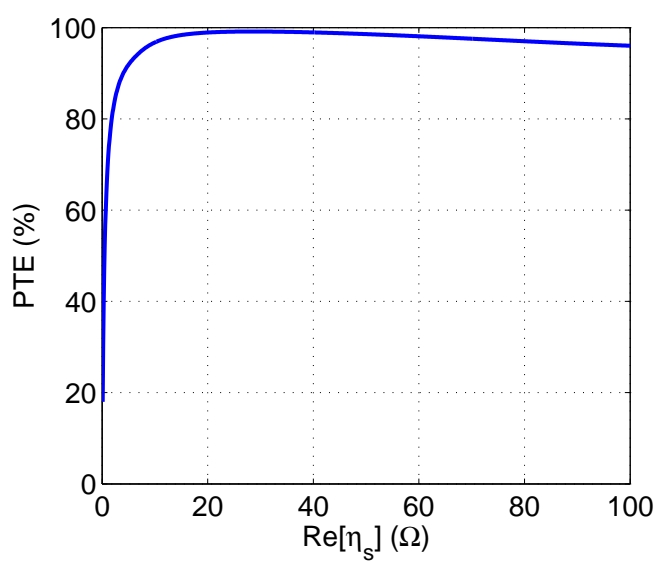

(b)

Fig. 13. (a) Power ratios and (b) power transfer efficiency PTE for the outof-phase $\left(\varepsilon_{c} \simeq-0.33\right)$ case as functions of the medium resistance, $\operatorname{Re}\left[\eta_{s}\right]$, in the metallic spherical core.

powers, Fig. 13a, as well as the PTE values, Fig. 13b, as the medium resistance in the metallic spherical core varies. For small values of that resistance, the out-of-phase case acts like the lossless case with $P_{\text {scat }}=P_{0}$. Then with the scattered field approximately out-of-phase with the source field, the radiated power is $14 \mathrm{~dB}$ smaller than $P_{0}$. When some dissipation is introduced into the scatterer, the out-of-phase case quickly yields a large $P T E$. Note, however, that $P_{\text {rad }}$ continues to be very small despite the presence of the dissipation. Moreover, the value of $P_{\mathrm{abs}}$ is smaller than in the resonance cases because of the limitation that the scattered power $P_{\text {scat }}=P_{0}$. Note also that when the losses are increased too much, they severely damp the scattering processes. This actually inhibits the destructive interference, which in turn reduces the $P T E$ values.

It is also worth remarking that large efficiencies are only achieved within the out-of-phase case at a very specific sourcescatterer separation distance. This is evidenced in Fig. 14, which presents the $P T E$ as a function of source-scatterer electrical separation distance, $k_{0} r^{\prime}$. To enable a comparison between the various resonant configurations, Fig. 14 provides the PTE curves for the same medium resistances that were considered for the dipolar resonance in Fig. 10. While the

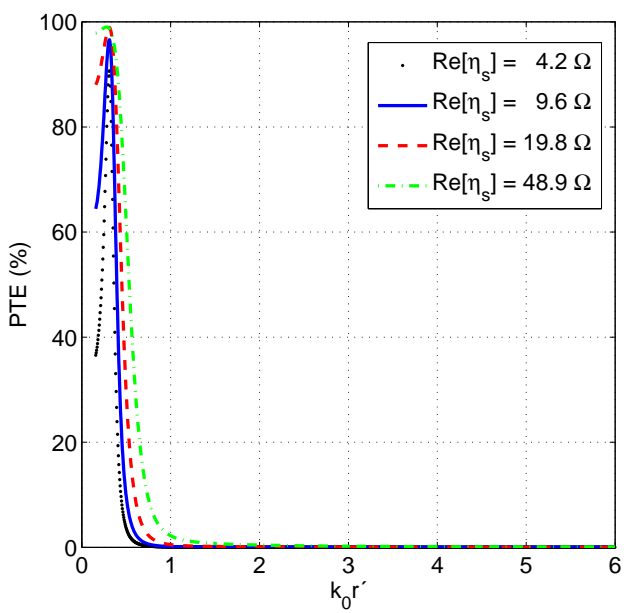

Fig. 14. Power transfer efficiency PTE for the out-of-phase case $\left(\varepsilon_{c} \simeq\right.$ $-0.33)$ as a function of the source-scatterer electrical separation distance, $k_{0} r^{\prime}$, for the medium resistances of the internal spherical core: $\operatorname{Re}\left[\eta_{s}\right]=$ $4.2 \Omega, 9.6 \Omega, 19.8 \Omega$ and $48.9 \Omega$

PTE monotonically decreases as the electrical separation distance increases, it is apparent from Fig. 14 that the PTE is maximized at a particular $k_{0} r^{\prime}$ value for these out-of-phase cases. This is due to the fact that the equality $P_{\text {scat }}=P_{0}$ must be ensured for the destructive interference to occur. Therefore, if the sensor is too close to the source, it is over-excited, i.e., $P_{\text {scat }}>P_{0}$; while if it is too far away, it is under-excited, i.e., $P_{\text {scat }}<P_{0}$.

To finalize our discussion, Fig. 15 illustrates how the balance of powers for the cloaking case $\left(\varepsilon_{c} \simeq 0.55\right)$ is affected by the presence of dissipation in the scatterer. Strikingly, a small increase in the medium resistance tunes the cloaking a bit better, i.e. it further reduces $P_{\text {scat }}$. This effect is due to the attenuation of the TE and higher-order TM modes (i.e., recall that only the TM dipolar mode is initially suppressed). However, in general both the $P_{\text {scat }}$ and $P_{\text {abs }}$ values increase along with the core's medium resistance. This is consistent with the fact that scattering cannot be completely suppressed in the presence of absorption. Note also that the cloaking point is less sensitive to losses than the other configurations. This occurs because it is not a resonant effect. Furthermore, Fig. 15 demonstrates that a substantial PTE $\simeq 35 \%$ can be obtained with an optimal amount of the losses, while $P_{\text {scat }}$ remains kept at a very low level: $P_{\text {scat }} \simeq-35 d B$. Consequently, the radiated power is that of the EHD source: $P_{\text {rad }} \simeq P_{0}$. Naturally, both $P T E$ and $P_{\text {abs }}$ are smaller than those achieved with resonant configurations. Despite this, it must be remarked that the cloaking case is a different paradigm. Although the scatterer is NF-coupled to the source and absorbing a significant amount of power, the outgoing radiation characteristics of the source remain unaltered. Under this perspective, a $P T E \simeq 35 \%$ value should be considered a remarkable performance.

This effect is further illustrated in Fig. 16, which presents the contour plot of the magnitude of the electric field along with the normalized total Poynting vector field, when the 


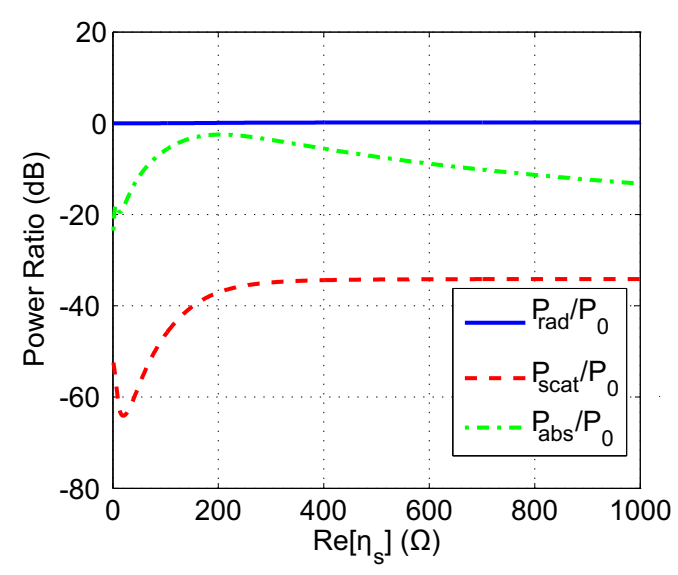

(a)

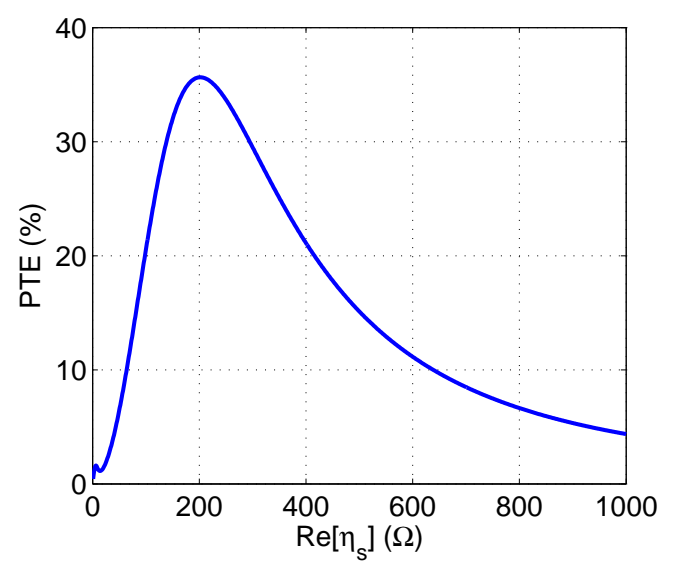

(b)

Fig. 15. (a) Power ratios and (b) power transfer efficiency $P T E$ at the cloaking point $\left(\varepsilon_{c} \simeq 0.55\right)$ as a function of the medium resistance, $\operatorname{Re}\left[\eta_{s}\right]$, in the metallic spherical core.

medium resistance of the metallic spherical core is $\operatorname{Re}\left[\eta_{s}\right]=$ $200 \Omega$. It can be concluded that, by using cloaked sensors such as those developed in [1], it is possible to efficiently transfer power in NF interactions, while maintaining a negligible disturbance of the radiation properties of the sources.

\section{CONCLUSIONS}

This work has presented a multipolar analysis of the absorption and scattering processes excited in near-field interactions. Closed-form expressions for the set of powers involved in these near-field interactions were given in a multipolar representation, (24)-(28). This set of equations enabled a direct evaluation of all of the involved powers, as well as the determination of the upper bounds of the powers of interest. Specifically, the maximum absorbed (30) and radiated (42) powers were determined. We found these limits of interest for the design of wireless power transfer and near-field parasitic radiator systems. Furthermore, the bounds were evaluated asymptotically in the high-frequency and quasi-static limits, corresponding to (49) and (50), respectively. The multipolar expressions allowed a more intuitive interpretation of the power bounds, as well as affording a means to identify the peculiarities of both the NF and FF interactions. Although the

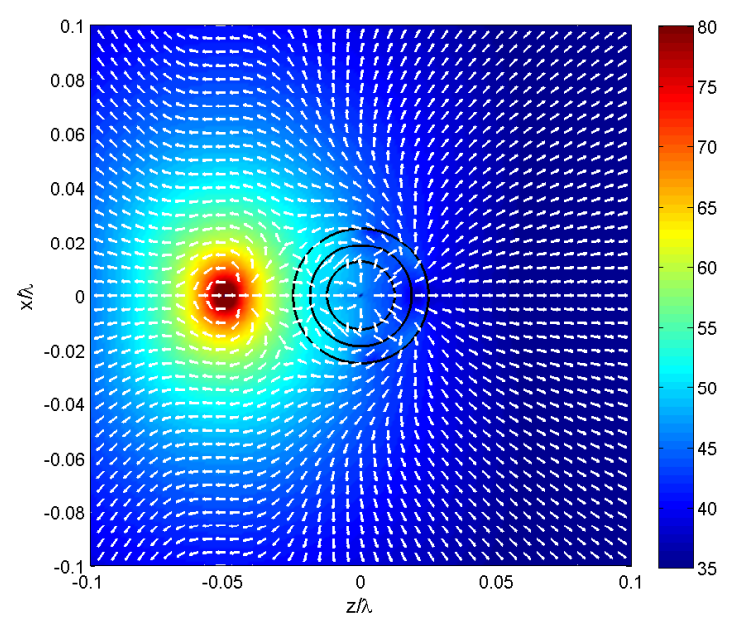

Fig. 16. [Color online] Contour plot of the magnitude of the total electric field, $10 \cdot \log _{10}\left|\mathbf{E}^{\mathrm{t}}\right|$, along with the normalized total Poynting vector field, $\mathbf{S}_{\text {tot }}$ at the cloaking point $\left(\varepsilon_{c} \simeq 0.55\right)$. The medium resistance of the metallic spherical core is $\operatorname{Re}\left[\eta_{s}\right]=200 \Omega$.

presented upper bounds were achieved with a discussion of ideal lossless antennas/scatterers, the presented formalism is also valid for lossy antennas/scatterers.

The analysis concluded that both the radiation and power transfer efficiencies can theoretically reach $100 \%$. While the total radiation efficiency is simply achieved with lossless materials, more complex strategies are needed to design highly efficient wireless power transfer systems. In this regard, two different strategies were studied: a scatterer radiating out-ofphase with the sources, and a scatterer with a large absorbedto-scattered power ratio. In the former case, the radiated power is suppressed by means of destructive interference occurring when the scatterer radiates out-of-phase with the sources. This directly leads to PTE $=100 \%$ as long there is absorption. In addition, it was shown to be theoretically possible to simultaneously obtain a suppression of the radiated power while keeping the absorbed power a maximum, as long as the combined multipolar condition (33) was satisfied. However, it was also demonstrated that no source can satisfy this condition in NF interactions. More restrictively, it also was demonstrated that dipolar sources cannot satisfy (33), no matter what the source-scatterer separation distance is. Thus, achieving a power transfer efficiency $P T E=100 \%$ by means of destructive interference necessitates a significant reduction of the absorbed power. Moreover, perfect destructive interference is only achieved for a very specific source-scattering configuration. As for the latter strategy, PTE $=100 \%$ can also be asymptotically achieved in NF interactions when the scatterer features a large absorbed-to-scattered power ratio. However, this situation also only comes at the cost of a decrease in the absorbed power. In fact, it has been demonstrated that the maximization of $P_{\text {abs }}$ imposes a $P T E=50 \%$ result in the NF case. All in all, it is concluded that there are inherent tradeoffs between $P T E$ and $P_{\text {abs }}$ in any NF-WPT system. One finds that PTE $=100 \%$ can be obtained in a NFWPT system if one sacrifices the PTE as the source-scatterer 
separation distance increases, the robustness of the system against undesired dissipation, and the speed of transfer.

Finally, the theoretical analyses and their conclusions were illustrated with a sensor example consisting of an electric Hertzian dipole illuminating a multi-layered sphere. This example was representative of electrically small devices and served to illustrate how the performance bounds can be approached under different phenomena. In particular, multipolar resonances, destructive interference and cloaking situations were discussed in detail. The practical challenge now is to design a sensor-parasitic resonator system that could mimic the performance characteristics of the multilayered spherical scatterer. This exercise is left to future efforts. It also was demonstrated that the multipolar resonances excited in the multilayered sphere can reach the performance limits of the absorbed and radiated powers. In addition, it was shown that the higher the multipole order, the larger $P_{\text {abs }}$ will be in both $\mathrm{NF}$ and FF interactions. In exchange, it also was found that the higher the multipole order, the smaller the bandwidth, the larger the transitory time to achieve the effects, and the more sensitive the associated resonance are to losses.

Further scattering cases confirmed that large PTEs are achieved when the coating layer forces the scatterer to radiate out-of-phase with the source. This condition suppresses the leakage of power from the system. However, the example also confirmed that the absorbed power is smaller than in the resonant cases and that the efficiency of the power transfer is restricted to very specific (and initially) selected sourcescatterer separation distances. Finally, a cloaked sensor was investigated. A different paradigm was confirmed in which the sensor is able to absorb an amount of power comparable to the power radiated by the sources, without altering either of their radiation properties.

\section{REFERENCES}

[1] A. Alù and N. Engheta, "Cloaking a sensor," Phys. Rev. Lett., vol. 102, no. 23, 233901, Jun. 2009.

[2] J. B. Andersen and A. Frandsen, "Absorption efficiency of receiving antennas," IEEE Trans. Antennas Propag., vol. 53, no. 9, pp. 28432849, Sep. 2005.

[3] D. H. Kwon and D. Pozar, "Optimal characteristics of an arbitrary receive antenna," IEEE Trans. Antennas Propag., vol. 57, no. 12, pp. 3720-3727, Dec. 2009.

[4] I. Liberal and R. W. Ziolkowski, "Analytical and equivalent circuit models to elucidate power balance in scattering problems," submitted to IEEE Trans. Antennas Propag., 2012.

[5] R. F. Harrington, "On the gain and beamwidth of directional antennas," IRE Trans. Antennas Propag., vol. 6, no. 3, pp. 219-225, 1958.

[6] _ Time-Harmonic Electromagnetic Fields. New York: McGrawHill, 1961.

[7] K. Y. Kim, Wireless Power Transfer - Principles and Engineering Explorations. InTech, 2012.

[8] R. W. Ziolkowski, P. Jin, and C. C. Lin, "Metamaterial-inspired engineering of antennas," Proc. IEEE, vol. 99, no. 10, pp. 1720-1731, Oct. 2011.

[9] V. N. Pustovit and T. V. Shahbazyan, "Resonance energy transfer near metal nanostructures mediated by surface plasmons," Phys. Rev. B, vol. 83, no. 8, pp. 5427-5431, Feb. 2011.

[10] A. S. Y. Poon, S. O'Driscoll, and T. H. Meng, "Optimal frequency for wireless power transmission into dispersive tissue," IEEE Trans. Antennas Propag., vol. 58, no. 5, pp. 1739-1750, May 2010.

[11] S. Kim, S. Member, J. S. Ho, A. S. Y. Poon, and S. Member, "Wireless power transfer to miniature implants: Transmitter optimization," IEEE Trans. Antennas Propag., vol. 60, no. 10, pp. 4838-4845, 2012
[12] J. Lee and S. Nam, "Fundamental aspects of near-field coupling small antennas for wireless power transfer," IEEE Trans. Antennas Propag., vol. 58, no. 11, pp. 3442-3449, Nov. 2010.

[13] Y. Tak and S. Nam, "Extended mode-based bandwidth analysis for asymmetric near-field communication systems," IEEE Trans. Antennas Propag., vol. 60, no. 1, pp. 421-424, Jan. 2012.

[14] A. Alù and S. I. Maslovski, "Power relations and a consistent analytical model for receiving wire antennas," IEEE Trans. Antennas Propag., vol. 58, no. 5, pp. 1436-1448, May 2010

[15] R. G. Newton, "Optical theorem and beyond," Am. J. Phys., vol. 44, no. 7, pp. 639-642, 1976.

[16] P. S. Carney, J. C. Schotland, and E. Wolf, "Generalized optical theorem for reflection, transmission, and extinction of power for scalar fields," Phys. Rev. E, vol. 70, pp. 6611-6617, Sep. 2004.

[17] D. S. Jones, The Theory of Electromagnetism. Pergamon, Oxford, 1965.

[18] R. Ziolkowski and A. Kipple, "Application of double negative materials to increase the power radiated by electrically small antennas," IEEE Trans. Antennas Propag., vol. 51, no. 10, pp. 2626-2640, Oct. 2003.

[19] S. Arslanagić, R. W. Ziolkowski, and O. Breinbjerg, "Analytical and numerical investigation of the radiation from concentric metamaterial spheres excited by an electric Hertzian dipole," Radio Sci., vol. 42, no. 6, RS6S16, Oct. 2007.

[20] A. Alù and N. Engheta, "Achieving transparency with plasmonic and metamaterial coatings," Phys. Rev. E, vol. 72, no. 1, 016623, Jul. 2005.

[21] _ _Polarizabilities and effective parameters for collections of spherical nanoparticles formed by pairs of concentric double-negative, singlenegative, and/or double-positive metamaterial layers," J. Appl. Phys. vol. 97, no. 9, 094310, 2005.

[22] R. Ziolkowski and A. Kipple, "Reciprocity between the effects of resonant scattering and enhanced radiated power by electrically small antennas in the presence of nested metamaterial shells," Phys. Rev. E, vol. 72, no. 3, 036602, Sep. 2005.

[23] A. Erentok and R. W. Ziolkowski, "Metamaterial-inspired efficient electrically small antennas," IEEE Trans. Antennas Propag., vol. 56, no. 3, pp. 691-707, Mar. 2008

[24] P.-Y. Chen and A. Alù, "Mantle cloaking using thin patterned metasurfaces," Phys. Rev. B, vol. 84, no. 20, pp. 205 110-205 112, Nov. 2011.

[25] C. A. Balanis, Advanced Engineering Electromagnetics. New York: Wiley, 2012.

[26] “NIST Digital Library of Mathematical Functions," http://dlmf.nist.gov/, Release 1.0.5 of 2012-10-01, online companion to [27]. [Online]. Available: http://dlmf.nist.gov/

[27] F. W. J. Olver, D. W. Lozier, R. F. Boisvert, and C. W. Clark, Eds., NIST Handbook of Mathematical Functions. New York, NY: Cambridge University Press, 2010, print companion to [26].

[28] T. V. Hansen, O. S. Kim, and O. Breinbjerg, "Stored energy and quality factor of spherical wave Functions - in relation to spherical antennas with material cores," IEEE Trans. Antennas Propag., vol. 60, no. 3, pp. 1281-1290, Mar. 2012.

[29] M. J. Berg, C. M. Sorensen, and A. Chakrabarti, "Extinction and the optical theorem. Part I. Single particles," J. Opt. Soc. Am. A, vol. 25, no. 7, pp. 1504-1513, Jul. 2008.

[30] S. D. Campbell and R. W. Ziolkowski, "Impact of strong localization of the incident power density on the nano-amplifier characteristics of active coated nano-particles," Opt. Commun., vol. 285, no. 16, pp. 3341-3352, Jul. 2012.

[31] S. Arslanagić and R. W. Ziolkowski, "Active coated nano-particle excited by an arbitrarily located electric Hertzian dipole - resonance and transparency effects," J. Opt., vol. 12, 024014, Jan. 2010. 


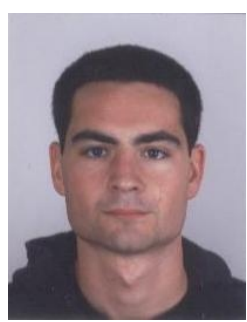

Iñigo Liberal was born on October 31, 1985 in Pamplona, Navarra, Spain. In 2009 he received the M. Sc. Degree in Telecommunication Engineering from the Public University of Navarra (UPNA), Pamplona, Spain. He was the recipient of a scholarship to carry out his M. Sc. Thesis at the International Research Center for Telecommunications and Radar (IRCTR) at the Delft University of Technology, The Netherlands. He is currently with the Antenna Group at the Public University of Navarra (UPNA), where he is working towards his Ph.D degree.

His main research interests are in the fields of applied electromagnetics and antenna theory, with emphasis on electromagnetic manipulation, artificial electromagnetic materials, and high-frequency applications of ferromagnetic wires.

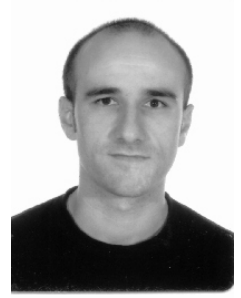

Iñigo Ederra was born in Isaba, Navarra, Spain in 1972. He received the Ingeniero de Telecomunicación and Ph.D. degrees from the Universidad Pública de Navarra, Pamplona, Spain, in 1996 and 2004, respectively.

In 1997, he joined the Microwave and Millimetre Wave Group, Universidad Pública de Navarra. From 1999 to 2000 he was with the European Space Research and Technology Centre (ESTEC), ESA, Noordwijk, The Netherlands, where he was working on Electromagnetic Bandgap materials and their applications in the field of antennas. Since 2001 he is with the Antenna Group, Universidad Pública de Navarra. From June to October 2002 he was visitor scientist at the Rutherford Appleton Laboratory, Chilton, Didcot, UK, participating in the Startiger project.

He was co-recipient of the LAPC 2006 and IWAT 2007 best paper award. His research interests are in the field of Electromagnetic Bandgap materials and Metamaterials and their applications in microwave and millimetre wave components and antennas.

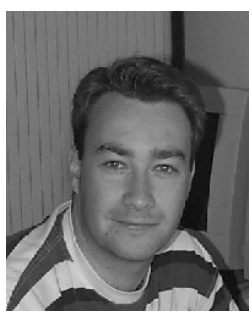

Ramón Gonzalo (S 95) was born on July 15, 1972 in Logroño, La Rioja. He received the M.Sc. and the $\mathrm{Ph}$. D. degree in Ingeniero de Telecomunicación both with honors, from the Public University of Navarra (UPNa), Spain.

Since October 1995 he is with the Antennas Group at the Electrical and Electronic Engineering Department in UPNa where he currently is Associate Professor. From September 1997 to December 1998, he was joined as Research Fellow to the Antenna Section in ESA-ESTEC where he was involved in the modelling and design of electromagnetic crystal devices at microwave and millimetre wave frequencies. He has been involved in more than 25 research project, European, and National level acting as co-ordinator in several of them. In particular he has been co-ordinator of 5 projects funded by the European Space Agency, 2 projects in the framework of the European Commission and several of them funded by the Spanish Minister of Science. He has more than 50 journal publications in peer review magazines and 100 conference papers related to his research lines. From January 2006 untill April 2008 he was acting as sub-director of the Engineering Faculty and from April 2008 he is acting as Head of Electrical and Electronic Engineering Department.

His current area of research is in the field of Terahertz technologies, subsystems and devices, Electromagnetic Band Gap technology with emphasis on space antenna applications, design of waveguide transmission lines and corrugated horn antennas.

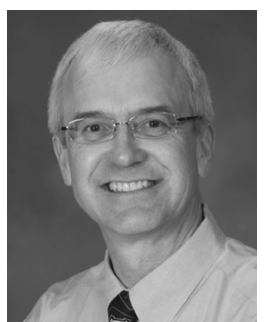

Richard W. Ziolkowski (M'87SM'91F'94) received his B.Sc. degree (hons) in physics (magna cum laude) from Brown University Providence, RI, USA, in 1974. and the M.S. and Ph.D. degrees in physics from the University of Illinois at UrbanaChampaign, IL, USA, in 1975 and 1980, respectively.

He was awarded an Honorary Doctorate, Doctor Technish Honoris Causa, from the Technical University of Denmark (DTU) in 2012. He was a member of the Engineering Research Division at the Lawrence Livermore National Laboratory from 1981 to 1990 and served as the leader of the Computational Electronics and Electromagnetics Thrust Area for the Engineering Directorate.He currently is serving as the Litton Industries John M. Leonis Distinguished Professor in the Department of Electrical and Computer Engineering at the University of Arizona. He holds a joint appointment with the College of Optical Sciences.

Prof. Ziolkowski is an OSA Fellow. He was the President of the IEEE Antennas and Propagation Society in 2005. He continues to be very active in the IEEE, OSA, and APS professional societies. His research interests include the application of new physics and engineering ideas to linear and nonlinear problems dealing with the interaction of electromagnetic waves with complex media, metamaterials, and realistic radiating and scattering structures. 